\title{
¿Velocity-Based EDR Retrieval Techniques Applied to Doppler Radar Measurements from Rain: Two Case Studies
}

\author{
A. C. P. OUdE NiJHuis \\ Electrical Engineering, Mathematics and Computer Science, Delft University of Technology, Delft, Netherlands \\ C. M. H. UNAL \\ Civil Engineering and Geosciences, Delft University of Technology, Delft, Netherlands \\ O. A. KRASNOV \\ Electrical Engineering, Mathematics and Computer Science, Delft University of Technology, Delft, Netherlands
}

\author{
H. W. J. RUSSCHENBERG
}

Civil Engineering and Geosciences, Delft University of Technology, Delft, Netherlands

\author{
A. G. YAROVOY
}

Electrical Engineering, Mathematics and Computer Science, Delft University of Technology, Delft, Netherlands

(Manuscript received 27 May 2018, in final form 8 June 2019)

\begin{abstract}
In this article, five velocity-based energy dissipation rate (EDR) retrieval techniques are assessed. The EDR retrieval techniques are applied to Doppler measurements from Transportable Atmospheric Radar (TARA) - a precipitation profiling radar-operating in the vertically fixed-pointing mode. A generalized formula for the Kolmogorov constant is derived, which gives potential for the application of the EDR retrieval techniques to any radar line of sight (LOS). Two case studies are discussed that contain rain events of about 2 and $18 \mathrm{~h}$, respectively. The EDR values retrieved from the radar are compared to in situ EDR values from collocated sonic anemometers. For the two case studies, a correlation coefficient of 0.79 was found for the wind speed variance (WSV) EDR retrieval technique, which uses 3D wind vectors as input and has a total sampling time of $10 \mathrm{~min}$. From this comparison it is concluded that the radar is able to measure EDR with a reasonable accuracy. Almost no correlation was found for the vertical wind velocity variance (VWVV) EDR retrieval technique, as it was not possible to sufficiently separate the turbulence dynamics contribution to the radar Doppler mean velocities from the velocity contribution of falling raindrops. An important cause of the discrepancies between radar and in situ EDR values is thus due to insufficient accurate estimation of vertical air velocities.
\end{abstract}

\section{Introduction}

Advanced weather radars have the potential to be used in civil applications with high-spatiotemporalresolution maps of rain, wind vectors, and turbulence.

¿ Denotes content that is immediately available upon publication as open access.

Corresponding author: A. C. P. Oude Nijhuis, albertoudenijhuis@ gmail.com
For example, rainfall rates can be estimated from X-band polarimetric radar measurements with a high spatial resolution (e.g., Anagnostou et al. 2004). It is expected that, when the measurement techniques are optimized, the advanced weather radar can deliver useful wind and turbulence-intensity products to enhance wind-hazard awareness. They also have the potential to measure wind dynamics in an all-weather monitoring system of wake vortex hazards at airports (e.g., Oude Nijhuis et al. 2018). However, the measurement of wind dynamics (wind vector and turbulence intensity) from backscattered 
electromagnetic waves from precipitating raindrops via the Doppler effect leads to questions such as whether sufficiently accurate results can be achieved. This will be further explained.

Turbulence is one of the most important physical processes in the atmospheric boundary layer (ABL). Knowledge of turbulence in the ABL is important for understanding vertical transport of momentum, mass, water vapor, enthalpy, and air pollutants (e.g., Garratt 1992; Pope 2000). It is also important in the formation and evolution of clouds and precipitation (Albrecht et al. 2016; Khain et al. 2015; Borque et al. 2016). Better understanding of cloud microphysical processes can lead to improved NWP and global climate models (Boutle and Abel 2012).

The turbulent energy dissipation rate (EDR), sometimes called eddy dissipation rate (Nastrom and Eaton 1997; Chan 2011), is a measure that indicates the intensity of turbulence (e.g., Pope 2000). More specifically, EDR is a measure of the viscous diffusion of turbulent kinetic energy (TKE), which is transformed from large scales to small scales within the inertial subrange via eddies, where eventually viscous effects dominate (Kolmogorov 1941). EDR is also a key parameter in large-eddy simulations (LESs), where it is used to close the momentum equation, to simulate ABLs with realistic Reynolds numbers (Meneveau and Katz 2000). Such advanced LES models can also be used in combination with a radar simulator to develop and validate EDR retrieval techniques, for example, with an X-band radar during clear-air conditions (Kovalev et al. 2016).

For many years, EDR values have been derived from in situ measurements. Close to the surface, EDR is typically derived from three-component sonic anemometers and/or fine platinum wire thermometers mounted on towers (e.g., Kaimal et al. 1976; Caughey et al. 1979; Zhou et al. 1985; Piper and Lundquist 2004). At higher altitudes, EDR is estimated from gust wind probe systems mounted on aircraft (e.g., Nicholls 1978; Lemone and Pennell 1979; Brost et al. 1982; Nucciarone and Young 1991), or from sonic anemometer carried by balloonborne sondes (e.g., O'Connor et al. 2010). Next to deriving EDR from velocity or temperature fluctuations, it can be estimated from other atmospheric variables that are indirectly influenced, such as the atmospheric refractive index (e.g., Thiermann and Grassl 1992).

More recently, active remote sensors, such as profiling Doppler radars and lidars, have been used to estimate EDR (Bryant and Browning 1975; Kollias and Albrecht 2000; Kollias et al. 2001; Meischner et al. 2001; O'Connor et al. 2010; Shupe et al. 2012; Röhner and Träumner 2013; Fang et al. 2014; Borque et al. 2016).
Doppler lidars are able to measure air motions below the cloud base or in clear-sky conditions (Lamer and Kollias 2015). In the case of clouds, profiling cloud radars can be used to continue profiling of measurements beyond the cloud base. The combination of a profiling Doppler lidar and a profiling Doppler cloud radar can then, for example, be used to deliver a continuous turbulence intensity profile (Borque et al. 2016). Active remote sensing of EDR has been proven to be valuable, for measurements of clear sky and clouds, by comparison to independent measurements (O'Connor et al. 2010; Shupe et al. 2012).

In aviation, EDR is used as an indicator for turbulence hazards. For example, EDR can be estimated from vertical accelerations of aircraft, or virtually any device moving through air (MacCready 1964). In recent years, there is an increasing interest to exploit lidar and radar measurements at airports to estimate the EDR. For example at the Hong Kong international airport, operational turbulence monitoring is done by retrieving the EDR field with a scanning lidar (Chan 2011). Currently, wake vortex monitoring systems are under development, where EDR has the potential to be used as an input parameter (Gerz et al. 2005; Barbaresco et al. 2013). It is necessary to mention that not only high but also low EDR values can be hazardous for aviation. Under certain atmospheric conditions, very light or negligible turbulence plays an important role as it favors long-lasting wake vortices (e.g., Holzäpfel et al. 2003). When the EDR is accurately measured, it can be used to predict the lifetime of such wake vortices (Holzäpfel 2006). Typically, the accuracy of radar-based or lidarbased EDR retrieval is limited to only a classification of the turbulence intensity: heavy $\left(2.5 \times 10^{-2} \mathrm{~m}^{2} \mathrm{~s}^{-3}\right)$, moderate $\left(1.9 \times 10^{-3} \mathrm{~m}^{2} \mathrm{~s}^{-3}\right)$, and light $(1.3 \times$ $\left.10^{-4} \mathrm{~m}^{2} \mathrm{~s}^{-3}\right)$. The given typical EDR values in brackets were adapted from MacCready (1964). The equivalent typical values for $\operatorname{EDR}^{1 / 3}$ are heavy $\left(0.29 \mathrm{~m}^{2 / 3} \mathrm{~s}^{-1}\right)$, moderate $\left(0.12 \mathrm{~m}^{2 / 3} \mathrm{~s}^{-1}\right)$, and light $\left(0.05 \mathrm{~m}^{2 / 3} \mathrm{~s}^{-1}\right)$. The desire is to improve the accuracy beyond such a classification, and estimate EDR with an uncertainty margin. Improved estimation of EDR in the terminal control area (TMA) of airports can improve aviation efficiency and safety (Barbaresco et al. 2014, 2016). In particular the estimation and reduction of uncertainty of EDR in critical areas, such as aircraft takeoff and approach flight paths, is important for the improvement of wake vortex monitoring systems (Barbaresco et al. 2013; Thobois et al. 2015).

Radars and lidars have the ability to work operationally in a complementary way in different weather conditions like fog, precipitation, and dry air. For the observation of air motion, both instruments are relying 


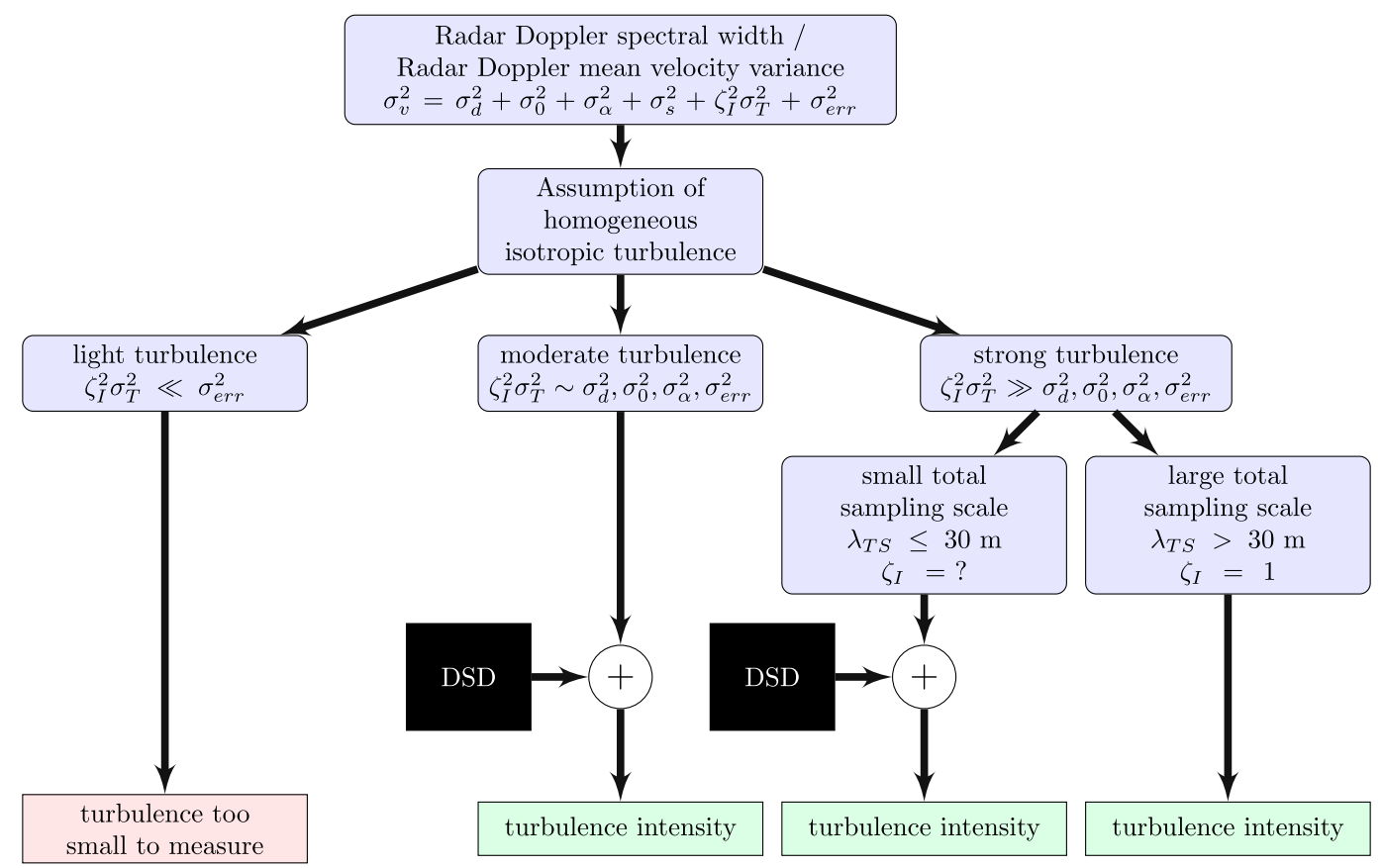

FIG. 1. The schematic illustrates the assumptions and simplifications that are used in radar-based turbulence intensity retrieval techniques. See Eq. (1) for the symbols used in this schematic.

on the backscatter of particles, which randomly fill the observation volume and are involved in air motion. Typical backscatterers for radar are rain/cloud drops and ice crystals, and aerosols for lidar. Different scattering and attenuation mechanisms of light/infrared waves for lidar and of microwaves for radar result in the fact that the lidar is able to retrieve the EDR remotely during clear-sky conditions, and the radar is able to do the same in presence of clouds or precipitation (Doviak and Zrnić 1993; Bringi and Chandrasekar 2001; Mishchenko et al. 2002). A difficulty with the radar to measure EDR with a Doppler radar during rain is then to take into account that raindrops are not perfect tracers of the air motion.

With a radar the Doppler spectral width, or the standard deviation of a series of Doppler velocities, $\sigma_{v}$ is measured, which can be modeled as a summation of independent terms as

$$
\sigma_{v}^{2}=\sigma_{d}^{2}+\sigma_{0}^{2}+\sigma_{\alpha}^{2}+\sigma_{s}^{2}+\zeta_{I}^{2} \sigma_{T}^{2}+\sigma_{\mathrm{err}}^{2},
$$

where $\sigma_{d}$ is due to variety in hydrometeor fall speeds, $\sigma_{0}$ is due to variety in hydrometeor orientations and vibrations, $\sigma_{\alpha}$ is due to antenna motion, $\sigma_{s}$ is due to shear, $\sigma_{T}$ is due to turbulence, $\zeta_{I}$ is a hydrometeor inertia correction, and $\sigma_{\text {err }}$ is an error due to model assumptions and/or measurement noise (Doviak and Zrnić 1993; Oude Nijhuis et al. 2016). Under special circumstances, additional terms can exist in Eq. (1), such as gravity waves (e.g., Nastrom 1997; Nastrom and Eaton 1997; Wilson 2004). The shear term can be retrieved from estimates of the wind vector derivatives (Gossard 1990; Borque et al. 2016). Precipitating raindrops have a nonnegligible terminal fall velocity, because of their relatively large mass and volume. Therefore, a significant contribution in Eq. (1) due to variety in hydrometeor fall velocities $\sigma_{d}$ can be expected. In addition, the raindrop inertia effect, which can be characterized as velocity differences due to relaxation, can enlarge or reduce the measured variance of turbulence velocities via $\zeta_{I}$ (Oude Nijhuis et al. 2016). The terms that are related to the rain characteristics, $\sigma_{d}$ and $\zeta_{I}$, can be estimated from radar observables via the raindrop size distribution (DSD) parameters. The rain DSD parameters can be estimated from the radar reflectivity, and/or other radar observables (e.g., Marshall and Palmer 1948; Brandes et al. 2004; Unal 2015).

The relative sizes of the terms in Eq. (1) determine how complex a radar-based turbulence intensity retrieval technique becomes. This is demonstrated in a schematic in Fig. 1. In this schematic the turbulence intensity is qualified by the relative magnitude of the turbulence term in comparison to the other terms in Eq. (1). When the turbulence term is smaller than the error term (relative light turbulence), the radar is not able to measure the turbulence intensity. To identify 
when this is occurring, a minimal value for turbulence intensity that can be measured with the radar can be estimated. When the turbulence term is larger than the error term, the turbulence intensity can be estimated but will rely on corrections for the other terms.

The rain DSD and the spatial scales of the measurements have an important role in the radar-based turbulence intensity estimation. The total sampling scale $\lambda_{\mathrm{TS}}$ is a measure of the spatial scale at which the velocity measurements are taken to calculate the standard deviation of velocities $\sigma_{v}$, and is used in the calculation of EDR. When EDR is estimated from the radar Doppler spectral width, the total sampling scale can be roughly estimated as $\lambda_{\mathrm{TS}}=V^{1 / 3}$, where $V$ is the total volumetric area that is illuminated in a single radar cell. A more refined expression that takes the power distribution pattern of the radar into account is given by White et al. (1999). The turbulence contribution to the Doppler spectral width, $\sigma_{T}$ [Eq. (1)], increases approximately with the total sampling scale $\lambda_{\mathrm{TS}}$ as $\sigma_{T} \propto \lambda_{\mathrm{TS}}^{1 / 3}$ in the inertial range because of the Kolmogorov $-5 / 3$ power law [e.g., Eq. (3.5) in White et al. 1999]. In this work it is assumed that the nonturbulent terms do not depend on the total sampling scale. Therefore, one solution to reduce the bias in EDR due to inaccurate information on the DSD is to enlarge the total sampling scale $\lambda_{\text {TS }}$ by using multiple radar cells for the estimation of velocity variance due to turbulence.

In the case that $\sigma_{d}$ is comparable to $\zeta_{I} \sigma_{T}$ (relative moderate turbulence), the DSD parameters are necessary to estimate the turbulence intensity. In the case that $\sigma_{d}$ is negligible compared to $\zeta_{I} \sigma_{T}$ (relative strong turbulence), it depends on the total sampling scale $\lambda_{\mathrm{TS}}$ whether the influence of the DSD has to be take into account. The exact value for "a sufficiently large total sampling scale to neglect the influence of measuring from raindrops" depends on the characteristics of the rain, where in general it can be stated that larger raindrops have a larger impact on the raindrop inertia term $\zeta_{I}$. The influence of raindrop inertia can be neglected for radar-based turbulence intensity retrieval techniques for all raindrop DSDs when the total sampling scale is larger than $30 \mathrm{~m}$ (Oude Nijhuis et al. 2016). Considering all effects of measuring EDR during rain with a radar, the turbulence intensity may be too small to measure, may need additional assumptions on the rain DSD, or can be derived without taking into account the influence of raindrops.

In the radar-based estimation of EDR, it is assumed that turbulence is homogeneous and isotropic, and the measurements are in the inertial range, until it is eventually dissipated to heat at the dissipation scale.
An idealized energy spectrum is assumed, having an extensive inertial range: The energy production takes places at the scale of weather systems, which is in the end via eddies dissipated to heat. This means that the energy production scale is approximately $1-10 \mathrm{~km}$, depending on the meteorological conditions, and the energy dissipation occurs at approximately the millimeter scale. In between the energy production scale and the dissipation scale, there is the inertial range where energy is converted to smaller and smaller scales via eddies. A schematic that illustrates the transfer of energy in the inertial range via eddies is presented in Fig. 2. As the dissipation takes place at the millimeter scale (e.g., Pope 2000; Piper and Lundquist 2004), this scale is irrelevant to weather radar measurements of turbulence intensity. For many EDR retrieval techniques, the measurements are assumed to be in the inertial range of the energy spectrum in Fig. 2. It should be stressed that this assumption of measuring in the inertial range of the energy spectrum has to be validated, as the turbulent kinetic energy spectrum shown in Fig. 2 is just a model.

Next to large-scale weather systems, there are many atmospheric phenomena at smaller scales that produce energy, such as frontal systems, building-induced turbulence, or surface friction. At airports, aircraft wake turbulence can be relevant (e.g., Frech 2007). For the cases where the measurements are not in the inertial range, it can be expected that the estimated EDR values are biased. Such cases should somehow be detected in an ideal EDR retrieval technique, and be accounted for in a radar-based estimation of EDR.

A correction for the influence of measuring from raindrops in radar-based EDR retrieval technique is challenging, and only useful for some specific scales given the rain DSD. A solution on how to account for the influence of raindrop inertia in radar-based turbulence intensity retrieval techniques is given in Oude Nijhuis et al. (2016), where an inertia correction model is proposed using inertial parameters based on the equations of motion for a raindrop and tuning parameters estimated from turbulence simulations.

To account for the inertia of scatterers, the DSD parameters have to be known, which can be based on the radar observables. Such a correction for raindrop inertia allows to resolve for a bias in EDR when it is not too dominant $\left[\zeta_{I}\right.$ close to 1 in Eq. (1)]. A large correction for raindrop inertia $\left(\zeta_{I}>2\right)$ becomes ineffective, as the uncertainty increases with the stochastic nature of turbulence (Oude Nijhuis et al. 2016). In this article, we do not use a correction for the influence of raindrop inertia $\left(\zeta_{I}=1\right)$ as the focus is mainly on EDR retrieval 


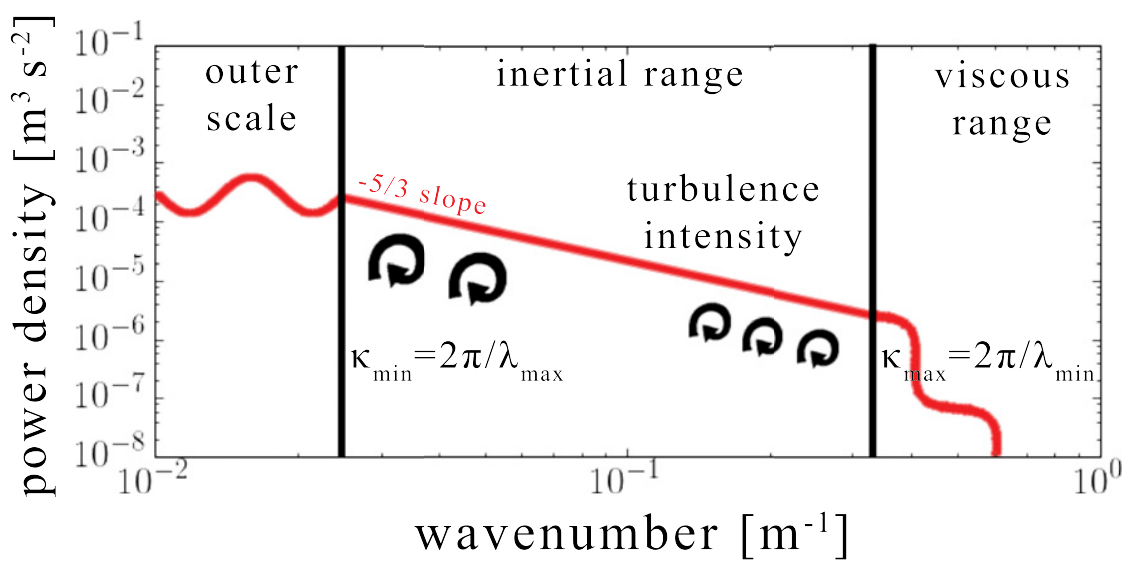

FIG. 2. In this schematic the theoretical power density of the energy spectrum is plotted against the wavenumber $k$. The outer scale provides the energy that is eventually dissipated to heat in the viscous range via eddies. In the middle, we find the inertial range, where energy is dissipated from larger to smaller scales, having a $-5 / 3$ power law according to Kolmogorov's hypothesis (Kolmogorov 1941).

techniques that have total sampling scales larger than $30 \mathrm{~m}$ (Oude Nijhuis et al. 2016).

The goal of this article is to apply a set of five velocitybased EDR retrieval techniques to radar-based estimated wind velocities during rain, and prove that the radar is capable of measuring turbulence under rainy conditions. The novelty lies in the active remote sensing of EDR during rain, which can be seen as an extension to other works, which applied such techniques during clear, cloudy, and/or drizzle conditions (e.g., Bryant and Browning 1975; Kollias and Albrecht 2000; Kollias et al. 2001; O'Connor et al. 2010; Shupe et al. 2012; Röhner and Träumner 2013; Fang et al. 2014; Borque et al. 2016).

To achieve this goal, a few velocity-based EDR retrieval techniques are selected, which can be applied to both in situ velocity measurements as well as remote radar Doppler measurements. Consequently, EDR values are compared to qualify the performance of these EDR retrieval techniques when applied to the radar during rainy conditions. In this work, the emphasis is on the application of EDR retrieval techniques with a total sampling scale larger than $30 \mathrm{~m}$, for which it is expected that the influence of raindrop inertia can be neglected. Energy spectra from collocated sonic anemometers are analyzed to validate that the measurements are in the inertial range. The EDR retrieval techniques are applied to measurements from Transportable Atmospheric Radar (TARA) (Heijnen et al. 2000), which is a precipitation profiling Doppler radar at the Cabauw meteorological supersite (Van Ulden and Wieringa 1996). This site contains a $200-\mathrm{m}$ tower, with sonic anemometers located at tower levers, which can be used for in situ validation of EDR. Two illustrative cases have been selected. One case study has relative high EDR values during a storm, whereas the other case has relative low EDR values.

This article is organized as follows. In section 2, a set of five velocity-based EDR retrieval techniques is described. In section 3, retrieved EDR values from the radar are validated with an in situ comparison. Conclusions are drawn in section 4 .

\section{Velocity-based EDR retrieval techniques}

In this section, an overview is given of a few velocity-based EDR retrieval techniques. When they are applied to the radar, a correction is applied for the terminal fall velocities of raindrops, based on the estimation of DSD parameters. The further specific details of the retrieval techniques are provided after this section.

As a consequence of Kolmogorov's similarity hypotheses, which are valid for homogeneous and isotropic turbulence, the turbulent energy spectrum $E(\kappa)$ of three-dimensional wind velocities in the inertial subrange is partitioned among the eddies in a universal form (Kolmogorov 1941; Sutton 1953; Pope 2000):

$$
E(\kappa)=C \varepsilon^{2 / 3} \kappa^{-5 / 3} \quad\left(\mathrm{~m}^{3} \mathrm{~s}^{-2}\right),
$$

where $\kappa=2 \pi / \lambda\left(\mathrm{m}^{-1}\right)$ is the wavenumber with length scale $\lambda(\mathrm{m}), \kappa$ is in the inertial range, $C=1.5$ is a Kolmogorov constant obtained from experiments (Pope 2000), and $\varepsilon\left(\mathrm{m}^{2} \mathrm{~s}^{-3}\right)$ is the EDR.

In addition to Eq. (2), there are similar expressions for the turbulent energy spectrum of the wind components, 
which are relevant for velocity measurements in the directions longitudinal or transverse to the main wind direction (e.g., Pope 2000). For application of turbulence retrieval techniques to a Doppler radar, it is convenient to be able to use any antenna-looking direction, or alternatively speaking, any line of sight (LOS). Therefore, the turbulent energy spectrum is formulated for any arbitrary LOS $E_{\mathrm{LOS}}$ :

$$
E_{\mathrm{LOS}}(\kappa)=C_{\mathrm{LOS}} \varepsilon^{2 / 3} \kappa^{-5 / 3},
$$

where $C_{\mathrm{LOS}}$ is the Kolmogorov constant for the LOS, given by

$$
\begin{aligned}
C_{\mathrm{LOS}}\left(\theta, \Delta_{\phi}\right)= & \cos ^{2} \theta \cos ^{2} \Delta_{\phi} C_{\mathrm{LL}}+\cos ^{2} \theta \sin ^{2} \Delta_{\phi} C_{\mathrm{TT}} \\
& +\sin ^{2} \theta C_{\mathrm{TT}}
\end{aligned}
$$

where $\theta$ is the radar antenna elevation angle, and $\Delta \phi$ is the angle between the LOS and wind direction in the horizontal plane, given by

$$
\Delta_{\phi}=\phi-\phi_{0},
$$

where $\phi$ is the radar antenna azimuth, and $\phi_{0}$ is the angle of the horizontal wind direction. The longitudinal Kolmogorov constant $C_{\mathrm{LL}}$ and the transverse Kolmogorov constant $C_{\text {TT }}$ are given by (e.g., Pope 2000)

$$
\begin{aligned}
& C_{\mathrm{LL}}=\frac{18}{55} C \approx 0.49, \\
& C_{\mathrm{TT}}=\frac{4}{3} C_{\mathrm{LL}}=\frac{24}{55} C \approx 0.65 .
\end{aligned}
$$

For writing down the radar Kolmogorov constant, Eq. (4), isotropy is assumed, which results in zero shear stress spectra [see section 6.5.8 of Pope (2000) for more details]. Note that the formulation of Eq. (3) still satisfies the turbulent energy spectrum for the longitudinal direction $E_{\mathrm{LL}}$, as $E_{\mathrm{LOS}}=E_{\mathrm{LL}}$ for $\left(\theta=0, \Delta_{\phi}=0\right)$, and for the transverse direction as $E_{\mathrm{LOS}}=E_{\mathrm{TT}}$ for $\left(\theta=0, \Delta_{\phi}=\pi / 2\right)$ or $(\theta=\pi / 2)$.

An alternative representation of the turbulent energy spectrum is the model for the second-order structure function (SSF), which is defined for each spatial separation $r$ as (Pope 2000)

$$
\begin{aligned}
D_{2, *}(r) & \equiv\left\langle\left[x\left(r^{\prime}+r\right)-x\left(r^{\prime}\right)\right]^{2}\right\rangle, \\
& =4 C_{*}(\varepsilon r)^{2 / 3}\left(\mathrm{~m}^{2} \mathrm{~s}^{-2}\right),
\end{aligned}
$$

where $x$ is the $1 \mathrm{D}$ wind velocity component or the 3D wind speed, and $C_{*}$ is a Kolmogorov constant, either $C$ or $C_{\mathrm{LOS}}$. The averaging is done over all possible locations $r^{\prime}$. Next to the second-order structure function model, there are models for higher-order structure functions (Katul 1994; Pope 2000). In this work, the higher-order structure functions are not considered, as it can be expected that the application of higher-order statistics results in inaccurate EDR values, because the higher-order statistics have stricter requirements with respect to sampling errors.

The formulas for the turbulence energy spectrum, Eqs. (2) and (3), and SSF, Eq. (9), are relevant for the spatial domain. To apply them to a time series of measurements, it is necessary to convert the energy spectrum and the structure function into the time domain. Using the Taylor hypothesis of frozen turbulence, the formulas are modified (Taylor 1938). For the conversion of the energy spectrum, wavenumbers are replaced by angular frequencies $\chi=2 \pi / t\left(\mathrm{~s}^{-1}\right)$ via the relation $\chi=U_{0} \kappa$, where $U_{0}\left(\mathrm{~m} \mathrm{~s}^{-1}\right)$ is the average $3 \mathrm{D}$ ambient wind speed, and the same kinetic energy is maintained as

$$
E_{*}(\chi)=C_{*} \varepsilon^{2 / 3} \chi^{-5 / 3} U_{0}^{2 / 3} \quad\left(\mathrm{~m}^{2} \mathrm{~s}^{-1}\right)
$$

For the structure functions, space lags $r$ are replaced by time lags $t$ via the relation $r=U_{0} t$. The values for $\chi$ are obtained from the sampling time interval and the total sampling time, with the assumption that the measurements are performed in the inertial range.

In this work, EDR retrieval techniques are relying on a series of $3 \mathrm{D}$ wind speed/1D wind velocity measurements, which can be obtained remotely via the Doppler effect with a radar, or in situ with a sonic anemometer. An overview of these techniques is given in Table 1, and the details are provided in the next subsections.

The set of EDR retrieval techniques includes a reference technique, the vertical wind velocity variance (VWVV) technique, which is often used (e.g., Bouniol et al. 2004; O'Connor et al. 2010; Shupe et al. 2012). It is desirable to apply this VWVV technique to the radar to be able to make a better comparison with other works and understand the limitations of this technique with regard to the radar instrument. This technique can be modified into the wind speed variance (WSV) technique, by using the variance of $3 \mathrm{D}$ wind speeds instead of the variance of vertical velocities. For in situ velocity measurements it is expected that the estimated EDR values will be similar and unbiased for the WSV and VWVV techniques as this is consistent with isotropic turbulence theory (e.g., Pope 2000). Note that isotropic turbulence is already an assumption in the Kolmogorov model to satisfy Eq. (2). However, for remote radar measurements during rain, the resulting EDR values 
TABLE 1. Overview of EDR retrieval techniques that are used in this work, their abbreviations, the scale at which they are applied, and the essential input variables. In this work a long time scale of $10 \mathrm{~min}$ is used, and for a short time scale $5 \mathrm{~s}$.

\begin{tabular}{lcc}
\hline \hline $\begin{array}{c}\text { EDR retrieval } \\
\text { technique }\end{array}$ & $\begin{array}{c}\text { Total } \\
\text { sampling scale }\end{array}$ & Input variables \\
\hline VWVV & Long & $\begin{array}{c}\text { Standard deviation of vertical } \\
\text { wind velocities and the } \\
\text { average 3D wind speed } \\
\text { Standard deviation of full 3D } \\
\text { wind speeds and the average }\end{array}$ \\
WSV & Long & 3D wind speed \\
STWSV & Short & $\begin{array}{c}\text { Standard deviation of full 3D } \\
\text { wind speeds and the average }\end{array}$ \\
& & 3D wind speed \\
PS & Long & Series of 3D wind speeds \\
SSF & Long & Series of 3D wind speeds \\
\hline
\end{tabular}

from the WSV technique can be much more accurate than the VWVV technique, because the more dominant horizontal wind velocity components are not prone to inaccurate terminal fall velocity corrections.

To address the turbulence scale dependence in this work, two total sampling times are used: a long time scale of $10 \mathrm{~min}$ and a short time scale of $5 \mathrm{~s}$. The 10 -min time scale is a typically used value (e.g., Siebert et al. 2006). Changing only the time scale in the WSV technique leads to the short time wind speed variance (STWSV) technique. When the sampling for these techniques (WSV and STWSV) with different sampling scales occurs in the inertial range, it is expected that they result in unbiased and similar EDR values. A large difference in EDR due to the applied total sampling time (short/long) can on the other hand thus be seen as an indication that the assumption of measuring isotropic turbulence in the inertial range is not satisfied.

In addition to the given techniques, we also consider a few other techniques, that use the SSF or the power spectrum (PS) of the wind speeds/velocities series. They are in particular interesting as they give an alternative uncertainty estimate for the retrieved EDR value, based on differences between a model function and measurements.

The set of EDR retrieval techniques that is given here has been implemented in a Python module, which is publicly available online (https:/github.com/albertoudenijhuis/ edrlib). It contains all the necessary functions to apply the EDR retrievals. Documentation and worked examples are available that demonstrate the application of the EDR retrieval techniques described in this work.

\section{a. Terminal fall velocity correction}

To obtain the vertical air velocity from radar Doppler measurements during rain, it is necessary to correct for the terminal fall velocities of raindrops. First, assumptions are made on the used rain DSD model and raindrop terminal fall velocity. Consequently, the DSD parameters are estimated, and a correction for the radar mean terminal fall velocity can be applied.

A common way to describe the rain DSD is by assuming a generalized gamma distribution with three parameters $N_{0}, \mu$, and $\Lambda$ (e.g., Brandes et al. 2004):

$$
N(D)=N_{0} D^{\mu} \exp (-\Lambda D)
$$

where $N(D)\left(\mathrm{mm}^{-1} \mathrm{~m}^{-3}\right)$ is the number of particles with an equivolumetric drop diameter between $D$ and $D+d D, D(\mathrm{~mm})$ is the drop equivolumetric diameter, $N_{0}$ is an absolute factor determining the number of particles per unit volume, and $\Lambda\left(\mathrm{mm}^{-1}\right)$ and $\mu(-)$ are distribution shape parameters.

The terminal fall velocity component along the radar beam is equal to (Atlas et al. 1973)

$$
v_{f}(D)=\alpha-\beta \exp (-0.6 D), \quad D \geq 0.109,
$$

where $D(\mathrm{~mm})$ is the equivolumetric drop diameter, and the parameters are given by

$$
\alpha=9.65 \sin \theta\left(\mathrm{m} \mathrm{s}^{-1}\right), \quad \beta=10.3 \sin \theta\left(\mathrm{m} \mathrm{s}^{-1}\right),
$$

where $\theta$ is the radar antenna elevation angle.

The estimate for the radar mean terminal fall velocity $\overline{v_{f}}$ is obtained by the following integral:

$$
\overline{v_{f}}=\int_{0}^{\infty} v_{f}(D) N(D) \sigma(D) d D / \int_{0}^{\infty} N(D) \sigma(D) d D,
$$

where $\sigma(D)$ is the radar cross section (RCS). For the estimation of $\overline{v_{f}}$, the absolute factor $N_{0}$ of the DSD is not required, and regarding the RCS only the dependency with $D$ has to be known. For weather radars we can assume Rayleigh scattering, and then the RCS is proportional to $D^{6}$. The estimate for the radar mean Doppler terminal fall velocity then simplifies to

$$
\overline{v_{f}}=\alpha-\beta\left[\frac{\Lambda}{\Lambda+0.6}\right]^{\mu+7} \text {. }
$$

The estimated vertical air velocity is then obtained by subtracting the estimated radar mean fall velocity from the measured mean Doppler velocity.

The estimation of radar DSD parameters is a challenging task for which several approaches can be taken. They can be based on a subset of radar observables, such as radar reflectivity, differential reflectivity, specific differential phases (e.g., Brandes et al. 2004), Doppler 
spectral analysis (e.g., Unal 2015), multifrequency techniques (e.g., Meagher and Haddad 2006), or an adaptive approach that uses in situ DSD measurements for calibration (e.g., Lane et al. 2014). The radar mean terminal Doppler velocity can be estimated for most of these radar-based estimated DSDs, as long as the DSD is assumed to have a generalized gamma distribution.

For our case studies the DSD parameters are estimated with work from Marshall and Palmer (1948), because the polarimetric approach is not effective as the radar is vertically oriented, and there is a lack of additional in situ DSD measurements for calibration or validation. Although this approach is not the state of the art (with regards to the aforementioned references), applying this first-order correction will improve the results for the estimated vertical velocities.

\section{b. Variance techniques (WSV, $V W V V$, and $S T W S V$ )}

Three EDR retrieval techniques that use the variance of wind as input are explained here. To apply these techniques in the time domain, the Taylor hypothesis of frozen turbulence (Taylor 1938) is used, which requires an average ambient wind speed $U_{0}$. The variance of the $3 \mathrm{D}$ wind speed sample series (or the variance of a wind component velocity series due to turbulence) is equivalent to the integral of the power spectrum, Eq. (10). For the time domain that is

$$
\begin{aligned}
\sigma_{T}^{2} & =\int_{\chi_{S}}^{\chi_{\mathrm{TS}}} E(\chi) d \chi \\
& =\frac{3}{2} C_{*} \varepsilon^{2 / 3} U_{0}^{2 / 3}\left[\chi_{S}^{-2 / 3}-\chi_{\mathrm{TS}}^{-2 / 3}\right],
\end{aligned}
$$

where $\chi_{S}$ is the angular frequency of the sampling, and $\chi_{\mathrm{TS}}$ is the total angular frequency of the sampling. The angular frequency $\chi_{S}$ is related to the sampling time $t_{S}$ via $\chi_{S}=2 \pi / t_{S}$. And the total angular frequency $\chi_{\mathrm{TS}}$ is in the same way related to the total sampling time $t_{\mathrm{TS}}$. Here $\sigma_{T}^{2}$ is the $3 \mathrm{D}$ wind speed variance in the case of the WSV or STWSV techniques, and $\sigma_{T}^{2}$ is the $1 \mathrm{D}$ wind component vertical velocity variance in the case of VWVV. The EDR can then be found by solving Eq. (17) for $\varepsilon$ :

$$
\varepsilon=\left(\frac{3}{2} C_{*}\left[\chi_{S}^{-2 / 3}-\chi_{\mathrm{TS}}^{-2 / 3}\right]\right)^{-3 / 2} U_{0}^{-1} \sigma_{T}^{3} .
$$

A potential problem with the wind variance measurements is that because of noise or errors the turbulence can be too small to measure, and an accurate retrieval of EDR is impossible. This is in particular

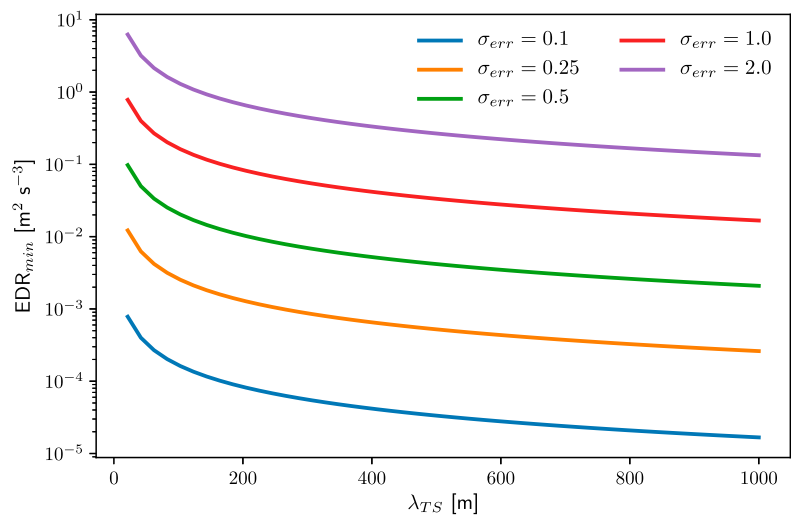

FIG. 3. Minimal retrievable EDR $\mathrm{EDR}_{\min }$ as a function of the total sampling scale $\lambda_{\mathrm{TS}}$ for different uncertainties in the variance of a series of wind velocities $\sigma_{\text {err }}\left(\mathrm{m} \mathrm{s}^{-1}\right)$. A Kolmogorov constant of $C=1.5$ is used, and the assumption is made of 50 equidistant samples $\left(\lambda_{\mathrm{TS}}=50 \lambda_{S}\right)$.

relevant for the radar, where there are contributions to the wind variance measurements that can be comparable to or even larger in size than the turbulence contribution [see Eq. (1)]. Given the error in the wind velocity standard deviation $\sigma_{\text {err }}$ [see Eq. (1)], a criterion for which EDR values cannot be accurately retrieved can then be formulated as

$$
\sigma_{T} \leq 2 \sigma_{\mathrm{err}} .
$$

In combination with Eq. (17), this results in a lower limit $\mathrm{EDR}_{\text {min }}$ for retrievable EDR, and is given by

$$
\mathrm{EDR}_{\min }=\left\{\frac{3}{2} C\left[\left(\frac{2 \pi}{\lambda_{S}}\right)^{-2 / 3}-\left(\frac{2 \pi}{\lambda_{\mathrm{TS}}}\right)^{-2 / 3}\right]\right\}^{-3 / 2}\left(2 \sigma_{\mathrm{err}}\right)^{3},
$$

where $C$ is a Kolmogorov constant, $\lambda_{S}=2 \pi / \kappa_{S}$ is the spatial sampling scale and $\lambda_{\mathrm{TS}}=2 \pi / \kappa_{\mathrm{TS}}$ is the total spatial sampling scale. The dependency of $\mathrm{EDR}_{\min }$ with the total spatial sampling scale $\lambda_{\text {TS }}$ is shown in Fig. 3. This figure demonstrates that by using a larger total sampling scale $\lambda_{\mathrm{TS}}$ the accuracy of EDR can be improved, in particular for light turbulence values. The assumption for this is that the measurements are in the inertial range [Eq. (2) applies], and that the other contributions to the wind variance measurement in Eq. (1) have no or weak dependencies on the total sampling scale.

In addition to the estimation of $\mathrm{EDR}_{\mathrm{min}}$, the uncertainty of retrieved EDR values for the variance techniques can be estimated with error propagation (e.g., Taylor 1997). Error propagation assumes uncorrelated and Gaussian distributed errors. As $\varepsilon$ varies on several orders of magnitude, this variable is not suitable for 
error propagation. The variable $\varepsilon^{1 / 3}$ is used here for the examination of the propagation of errors. Note that typical values for $\varepsilon$ and $\varepsilon^{1 / 3}$ are given in the introduction. For the time domain we obtain

$$
\begin{aligned}
\left(\frac{\sigma_{\varepsilon}}{\varepsilon^{1 / 3}}\right)^{2}= & {\left[\frac{1}{\varepsilon^{1 / 3}}\left(\partial \varepsilon^{1 / 3} / \partial \chi_{\mathrm{TS}}\right) \sigma_{\chi, \mathrm{TS}}\right]^{2} } \\
& +\left[\frac{1}{\varepsilon^{1 / 3}}\left(\partial \varepsilon^{1 / 3} / \partial \chi_{S}\right) \sigma_{\chi, S}\right]^{2} \\
& +\left[\frac{1}{\varepsilon^{1 / 3}}\left(\partial \varepsilon^{1 / 3} / \partial U_{0}\right) \sigma_{U 0}\right]^{2} \\
& +\left[\frac{1}{\varepsilon^{1 / 3}}\left(\partial \varepsilon^{1 / 3} / \partial \sigma_{T}\right) \sigma_{\sigma, T}\right]^{2} \\
= & \frac{1}{9} \frac{\left.\chi_{\mathrm{TS}}^{-10 / 3} \sigma_{\chi, \mathrm{TS}}^{2}+\chi_{S}^{-10 / 3} \sigma_{\chi, S}^{2}\right]}{\left[\chi_{\mathrm{TS}}^{-2 / 3}-\chi_{S}^{-2 / 3}\right]^{2}} \\
& +\frac{1}{9}\left(\frac{\sigma_{U_{0}}}{U_{0}}\right)^{2}+\frac{1}{9} \frac{9}{2(N-1)}, \\
\approx & \frac{1}{9}\left(\frac{\chi_{S}}{\chi_{\mathrm{TS}}}\right)^{4 / 3}+\frac{1}{9}\left(\frac{\sigma_{U_{0}}}{U_{0}}\right)^{2}+\frac{1}{2(N-1)},
\end{aligned}
$$

where $N$ is the number of samples, and $\sigma_{\chi, \mathrm{TS}}, \sigma_{\chi, S}, \sigma_{U_{0}}$, $\sigma_{\sigma, T}$ are the uncertainties of the variables in the subscript. The last approximation, Eq. (23), has been made by assuming that $\chi_{S} \ll \chi_{\mathrm{TS}}$. Here we used the relation $\sigma_{\sigma, T} / \sigma_{T}=[2(N-1)]^{-1 / 2}$ (Taylor 1997), and that $\sigma_{\chi} \approx \chi^{2} / \chi_{\mathrm{TS}}$. The last relation is derived from uncertainty propagation (e.g., Taylor 1997), $\left(\sigma_{\chi, \mathrm{TS}} / \chi_{\mathrm{TS}}\right)=\left(\sigma_{t, \mathrm{TS}} / t_{\mathrm{TS}}\right)$, and that $\sigma_{t, \mathrm{TS}} \approx t_{S}$, where $\sigma_{t, \mathrm{TS}}$ is the uncertainty in sampling time. From this uncertainty analysis, we conclude that sufficient samples are necessary for averaging $(N>50)$, the EDR estimation uncertainty increases with the relative variation of the wind speed $\sigma_{U_{0}} / U_{0}$, and the EDR estimation uncertainty increases with $\chi_{S} / \chi_{\mathrm{TS}}$. For reference we give the uncertainty propagation result for the space domain as well, for which the derivation is similar:

$$
\left(\frac{\sigma_{\varepsilon}}{\varepsilon^{1 / 3}}\right)^{2} \approx \frac{1}{9}\left(\frac{\kappa_{S}}{\kappa_{\mathrm{TS}}}\right)^{4 / 3}+\frac{1}{2(N-1)},
$$

where the assumption is made that $\kappa_{S} \ll \kappa_{\mathrm{TS}}$. We note here that this estimated uncertainty for EDR values should be treated carefully, and can be artificially low when the measurements are not in the inertial range, or the turbulence intensity fluctuates during the measurements (i.e., when the turbulence is not stationary). The estimated uncertainty of this and other methods will be further discussed for the measurements in the study cases.

\section{c. Techniques based on time series of wind speeds $(P S, S S F)$}

In this subsection, retrieval techniques are discussed where the EDR is obtained from the PS, or from the SSF. The assumption is that the measured velocity samples are in the inertial range of the energy spectrum. These two techniques allow for the estimation of EDR in sampling subdomains, and thus provide an alternative EDR uncertainty estimation.

For the power spectrum EDR retrieval technique (referred to as PS), first the power spectrum is calculated for a series of $3 \mathrm{D}$ wind speeds. The power spectrum is obtained via the Wiener-Khinchin theorem, where a discrete Fourier transformation is applied to the autocorrelation of the given samples. For each discrete frequency $\chi_{j}$ the power $P_{j}$ is calculated. Consequently, the spectrum is divided into a certain number of frequency intervals, and for each $i$ th interval $\left[\chi_{1}, \chi_{2}\right]$ we can find the standard deviation of wind speeds $\sigma_{i}$ :

$$
\sigma_{i}^{2}=\sum_{\chi_{1}}^{\chi_{2}} P_{j}
$$

Given $\sigma_{i}, \chi_{1}, \chi_{2}$, Eq. (18) is used to calculate an EDR value for the $i$ th frequency interval. The final estimate $\widehat{\varepsilon_{f}^{1 / 3}}$ comes from the average of $\varepsilon^{1 / 3}$ values:

$$
\widehat{\varepsilon_{f}^{1 / 3}}=\overline{\varepsilon^{1 / 3}} \pm \sigma_{\varepsilon},
$$

where the uncertainty in EDR is estimated from the standard deviation of $\varepsilon^{1 / 3}$ values.

To better comprehend the PS technique, we can look at the number of frequency intervals. In the limit of 1 frequency interval, the PS retrieval technique is the same as the WSV technique. This emphasizes the difference between the PS and WSV techniques. The WSV technique gives most of the weight of the retrieved EDR to the smallest frequencies (largest spatial/time scales), because of the $-5 / 3$ scaling with frequency in the spectrum [see Eq. (10)]. On the contrary, the PS technique with multiple frequency intervals gives extra weight to the higher frequencies (smaller spatial/time scales). For the PS technique in this work, the spectrum is divided into three equally sized frequency intervals. With this choice, the PS technique deviates from the WSV technique (just one interval), and at the same time unstable results are avoided that can occur with insufficient sampling.

The second-order structure function EDR retrieval technique (referred to as SSF) is applied in the time domain in this work. A time series of wind speeds with $N$ samples is used to calculate the structure function 


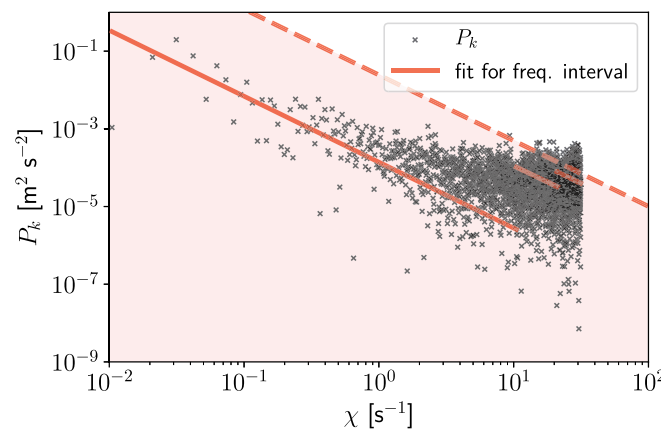

(a) PS, case A

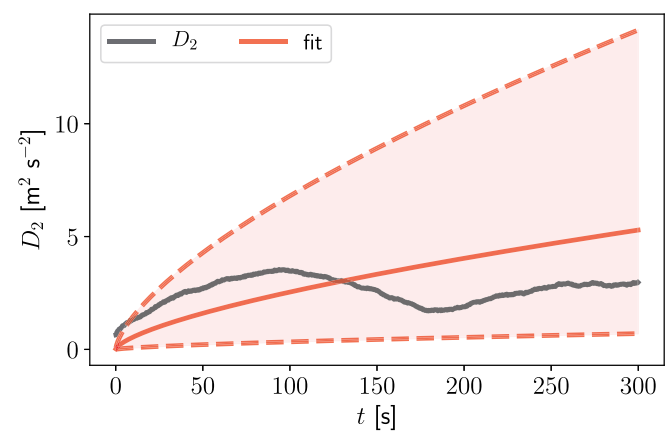

(c) SSF, case A

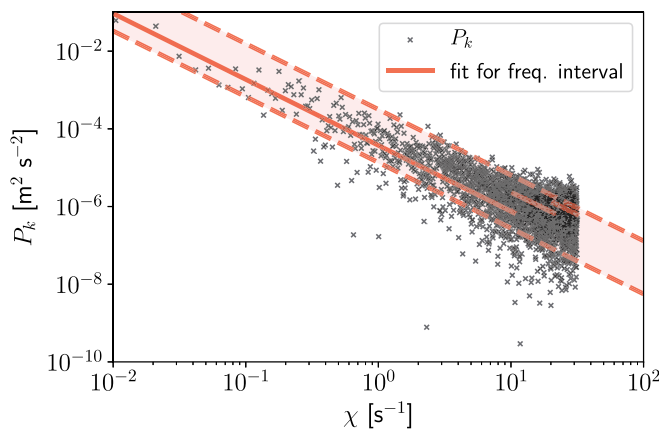

(b) PS, case B

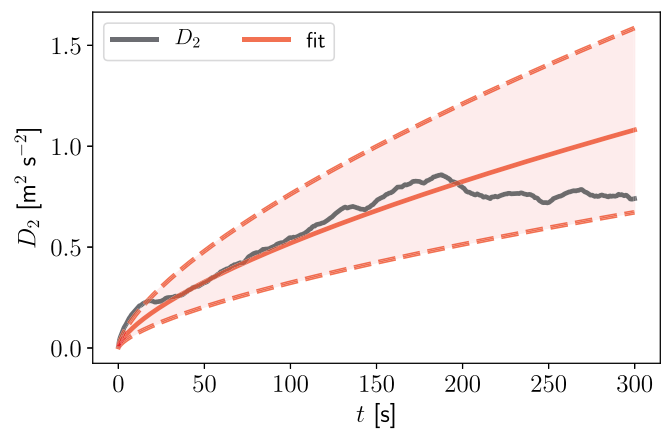

(d) SSF, case B

FIG. 4. Examples of the (a),(b) PS and (c),(d) SSF retrieval techniques for EDR, which have a series of 3D wind speeds as input. In (a),(b) the discrete power $P_{k}\left(\mathrm{~m}^{2} \mathrm{~s}^{-2}\right)$ is plotted as a function of angular frequency $\chi\left(\mathrm{s}^{-1}\right)$. In (c),(d) the second-order structure function $D_{2}\left(\mathrm{~m}^{2} \mathrm{~s}^{-2}\right)$ [Eq. (9)] is plotted as a function of lag time $t$ (s). The black crosses/lines show the processed velocity samples, and the red lines show the model functions. The dashed red lines show the 2 times STD uncertainty of the model (model function evaluated at $\overline{\varepsilon^{1 / 3}} \pm 2 \sigma_{\varepsilon}$ ). $3 \mathrm{D}$ wind speed measurements from sonic anemometers were used in all these examples, which have a sampling time of $t_{S}=0.1 \mathrm{~s}$, and a total sampling time of $t_{\mathrm{TS}}=600 \mathrm{~s}$. Case A is for 0900 UTC $10 \mathrm{Apr} 2012$, and case B is for 1200 UTC 10 Apr 2012.

[Eq. (9) in combination with $r=U_{0} t$ ] from time lags $t=t_{S}$ until $t=N t_{S} / 2$, where $t_{S}$ is the sampling time. This is done in a such way that at least $N / 2$ samples are used to calculate each term (for nonperiodic signals). Consequently, for each time lag $t$ an EDR value is obtained with

$$
\varepsilon=\frac{1}{U_{0} t}\left[\frac{D_{2, *}(t)}{4 C_{*}}\right]^{3 / 2} .
$$

The final EDR value and its uncertainty are then calculated from the $\varepsilon^{1 / 3}$ values for each time lag $t$, via Eq. (26).

Advantages of these techniques, with respect to the variance techniques, are that 1) insight can be obtained from visual inspection of fitted functions with regard to processed measurements and 2) a model error can be estimated from the fitted results. A disadvantage is that the processing becomes more complex, and more retrieval settings have to be chosen, which can lead to unstable results. With unstable it is meant that the result can depend on the chosen parameters, such as the number of frequency intervals for the PS technique.

In this work 3D wind speeds are used as input for the PS and SSF techniques, because the aim is to find techniques for the radar during rain, which are less dependent on accurate DSD parameter estimation, and the 3D wind speeds are less prone to terminal fall velocity corrections. Alternatively, wind velocity components could be used.

Two cases are shown in Fig. 4 for sonic anemometer measurements. For the PS technique, the discrete power $P_{k}\left(\mathrm{~m}^{2} \mathrm{~s}^{-2}\right)$ is plotted versus angular frequency $\chi\left(\mathrm{s}^{-1}\right)$. The model [Eq. (17)] is plotted for the three frequency interval for which EDR is estimated. In addition to that, the estimated uncertainty is plotted [Eq. (17)] with dashed lines. For case A the uncertainty was rather large, and there was no lower limit for the uncertainty $\left(\widehat{\varepsilon_{f}^{1 / 3}}-2 \sigma_{e}<0\right)$. For case B the uncertainty was much less, which indicates that the samples are more likely in 
the inertial range. For the SSF technique, the estimated EDR for each time lag [Eq. (27)] and the model function [Eq. (9)] are shown. Also, for the SSF the uncertainty of the estimated EDR is plotted with dashed lines.

\section{Selected case studies}

The validation of EDR retrieval techniques is done by a comparison of EDR values from a Doppler radar during rain with EDR values from in situ measurements. In this study two instruments are used, which are TARA-an S-band precipitation profiling radar (Heijnen et al. 2000)—and sonic anemometers placed on levers at several altitudes of a $200-\mathrm{m}$ research tower in the Netherlands. These instruments are located on a meteorological supersite, which is well equipped for atmospheric boundary layer research (Van Ulden and Wieringa 1996; Casso-Torralba et al. 2008). On this site the TARA radar and the research tower are closely located, and can be used for the comparison and validation of the EDR retrieval techniques.

For the validation of EDR by comparison, two rain events are chosen on 10 April and 21 June 2012. The first case study has moderate turbulence levels, whereas the latter case has some peak values for turbulence intensity [turbulence intensity levels can be found in, e.g., ICAO (2007)].

On 10 April 2012, a cold front passes Cabauw during the morning. The daily total rainfall is $4.1 \mathrm{~mm}$, which is accumulated in approximately $9 \mathrm{~h}$. The wind is coming mainly from the south-southwest.

On 21 June 2012 a cold front passes over the site in the evening. The daily total rainfall is $14.1 \mathrm{~mm}$, which is accumulated in approximately $3 \mathrm{~h}$. The wind is coming mainly from the east-southeast. During the rain on this day, there was a summer storm, for which lightning strikes were reported.

\section{a. Sonic anemometers}

For the estimation of in situ EDR values, the Gill R3 sonic anemometers are used, which are mounted at levers at $5,60,100$, and $180 \mathrm{~m}$ on the Cabauw research tower. The instantaneous wind speed and direction are determined using the effect of the wind influence on the propagation time of acoustic pulses transmitted in opposite directions from two arms of the instrument. The raw data, including the three orthogonal wind components and the sonic anemometer temperature, are stored with a frequency of $10 \mathrm{~Hz}$.

The wind component measurement accuracy is less than $0.02 \mathrm{~m} \mathrm{~s}^{-1}$, where effects of tower mounting are not taken into account. The sonic anemometer measurements can be disrupted by the presence of water droplets

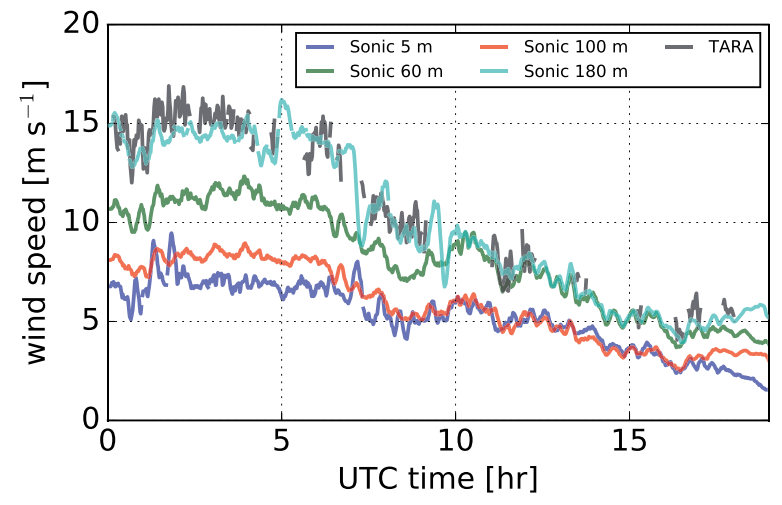

(a) 10 April 2012

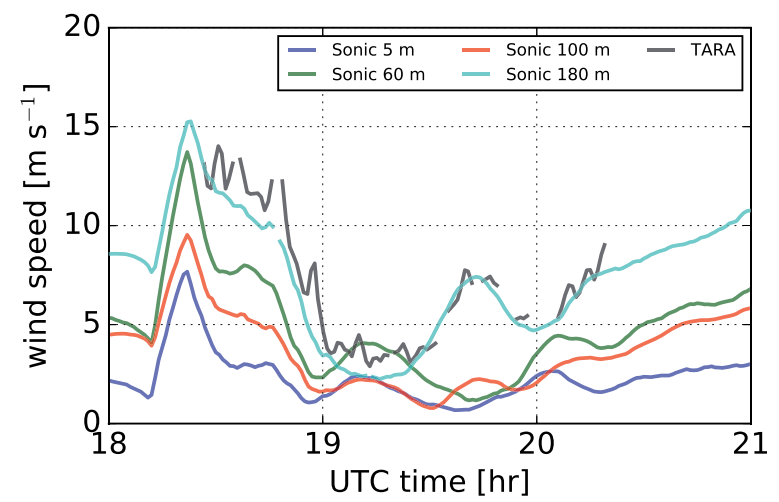

(b) 21 June 2012

FIG. 5. The 10-min moving average of wind speed on (a) $10 \mathrm{Apr}$ and (b) 21 Jun 2012 for the sonic anemometers at different altitudes and the first range cell of TARA where the radar resolution volume spans from 173- to 202-m altitude.

on the transducer. To mitigate this effect, we filter out data with unrealistic wind changes that are higher than $500 \mathrm{~m} \mathrm{~s}^{-2}$. Less than $1 \%$ is filtered by this procedure, and unrealistic velocity spikes during rain events are filtered out. Whenever the horizontal wind direction is in the range of $280^{\circ}-340^{\circ}$, the data quality is flagged, because for such cases the sonic anemometer is in the wake of the tower. For the calculation of moving averages we require to have at least $50 \%$ of the samples available within the total sampling time, and otherwise the data quality is flagged. Whenever the data quality is flagged, it is not used for comparisons or in plots.

Figure 5 shows measurements of the sonic anemometers wind speeds at different altitudes. We can see a typical vertical structure within these rain events for the wind speed, as it increases with height. In Fig. 6 the vertical wind velocity is shown for the sonic anemometer at $180-\mathrm{m}$ altitude. A 10 -min moving average is used to calculate the vertical wind velocity, which is on average close to zero and for the case studies never exceeds more than $2 \mathrm{~m} \mathrm{~s}^{-1}$ in magnitude. The 10 -min moving average 


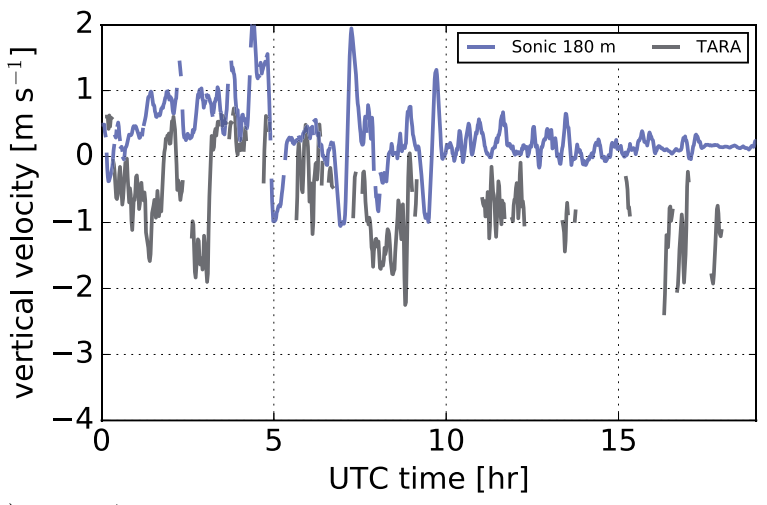

(a) 10 April 2012

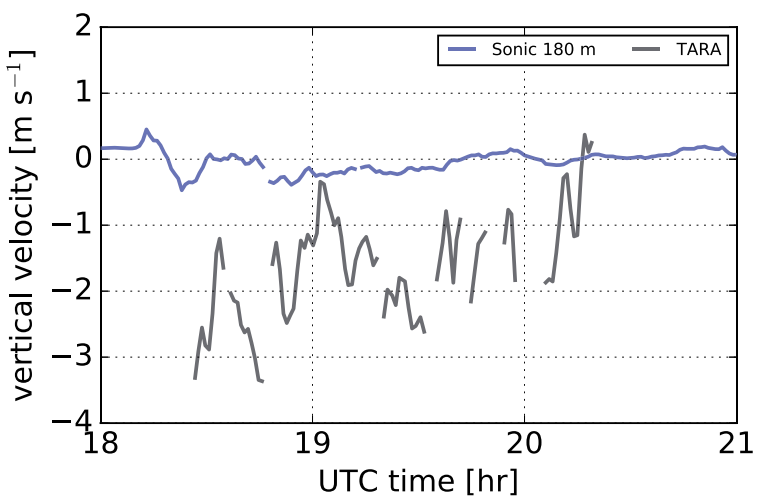

(b) 21 June 2012

FIG. 6. The 10-min moving average of vertical wind velocity measured with a sonic anemometer at $180-\mathrm{m}$ altitude and with TARA for the first range cell on (a) 10 Apr and (b) 21 Jun 2012. For TARA a correction has been applied for the raindrop terminal fall velocity [Eq. (15)].

for the vertical wind velocity is in particular useful for the validation of the radar-based vertical-velocity correction for raindrops. Using $10 \mathrm{~min}$ for averaging also overcomes difficulties of comparing the results from two instruments that are spatially separated. Figures 5 and 6 demonstrate thus that the sonic anemometers were capable to measure the vertical velocities during these rain events, and can thus be used for in situ validation. This is not trivial, because unrealistic spiked velocities had to be removed with the filtering strategy.

\section{b. TARA radar}

TARA is a polarimetric S-band (frequency $3.298 \mathrm{GHz}$, wavelength $9.1 \mathrm{~cm}$, horizontal/vertical polarizations, far-field region starting at $\sim 200 \mathrm{~m}$ range) frequencymodulated continuous wave (FMCW) radar that was developed at the Delft University of Technology (Heijnen et al. 2000). Besides the main beam with polarimetric measurement capabilities, TARA has two offset beams at vertical polarization pointing with an offset angle of $15^{\circ}$ with respect to the main beam in the radar antenna elevation-looking angle and the radar antenna azimuthlooking angle directions. Its antennas are pointing with a fixed antenna elevation $\left[\sigma_{\alpha}=0\right.$ in Eq. (1)]. In this work, the main beam has a radar antenna elevation-looking angle of $\theta=75^{\circ}$. The first offset beam has the same radar antenna azimuth-looking angle and an elevation-looking angle of $\theta=90^{\circ}$, and the second offset beam has a radar antenna azimuth-looking angle offset of $+15^{\circ}$, with a radar elevation-looking angle of $\theta=69^{\circ}$. The radar transmits linear frequency modulated sweeps, alternately at horizontal and vertical polarization for the main beam, and linear frequency modulated sweeps at vertical polarization for the two offset beams. The backscattered signal is received by a one-channel receiver, either from the main beam (horizontal or vertical polarization) or by one of the two offset beams.

The velocity estimation is based on analysis of the Doppler frequency shift, caused by the displacement of hydrometeor particles and spatial irregularities of atmospheric refractive index, which are related to variations in air temperature and humidity. A Doppler polarimetric dealiasing technique, described in Unal and Moisseev (2004), takes care of unfolding the Doppler velocities. By combining the Doppler measurements from the three beams, the horizontal wind speed and direction, and the vertical Doppler velocity can be estimated at high time and spatial resolution (Unal et al. 2012). For the estimation of the vertical air velocity, a correction for the raindrop terminal fall velocity is used (see section 3a).

The dataset contains the time-height indicators of the main beam reflectivity ( $\mathrm{dBZ}$ ), the estimated horizontal wind speed and direction and the vertical Doppler velocity. The processed radar data have 2.5-s time resolution and $30-\mathrm{m}$ range resolution. The altitude range is between $200 \mathrm{~m}$ and $15 \mathrm{~km}$. There is no radar data available from TARA in the case of clear-air and very calm conditions, because the measurement technique relies on backscattering from atmospheric inhomogeneities or particles. There is also no information when the LOS velocity modulus is below $\pm 0.2 \mathrm{~m} \mathrm{~s}^{-1}$, because of the Doppler filtering strategy in the nonpolarimetric offset beams.

In Fig. 7 the measured reflectivity factors and Doppler velocities for the main beam from TARA are shown. We can see that there was strong precipitation on 21 June 2012, as there were high reflectivity factors. On 10 April 2012 the rain intensities were moderate. For both cases, a melting layer can be recognized from increased reflectivity factors at $\sim 1.7$ and $\sim 3 \mathrm{~km}$, respectively. At these and higher altitudes, the interaction between the ambient wind and the tracers becomes much more 


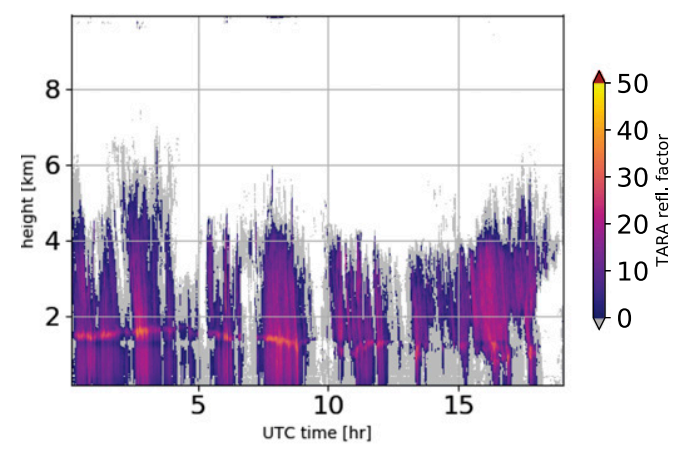

(a)

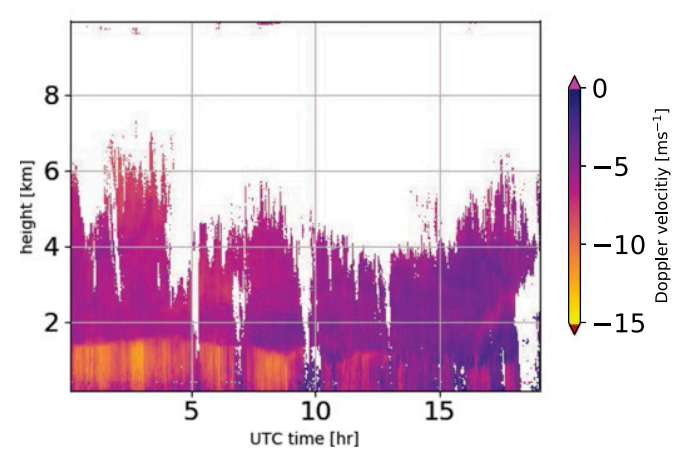

(c)



(b)

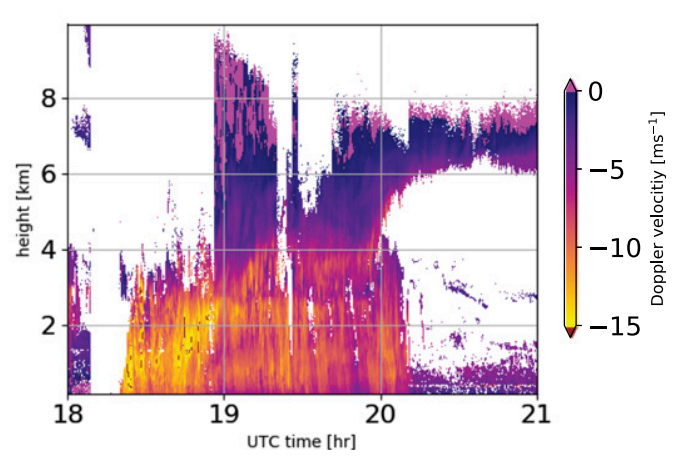

(d)

FIG. 7. Radar measurements from TARA: (a),(b) reflectivity factors and (c),(d) Doppler velocities for the main beam (antenna elevation angle of $75^{\circ}$ ) on (left) $10 \mathrm{Apr}$ and (right) 21 Jun 2012. Note that the measurement of the upper part of the precipitating cloud is missing before 1900 UTC in (b) and (d), because of the automatic decrease of the transmit power to avoid receiver saturation.

complex because of the presence of nonliquid hydrometeors, and is outside the scope of this study.

\section{c. Comparison of remote with in situ measurements}

In this work, only the first radar range cell of TARA is used for EDR estimation, because only at this altitude there is an in situ comparison possible with a sonic anemometer at $180-\mathrm{m}$ altitude. The first range cell of TARA has a sampling space that has its altitude between 173 and $202 \mathrm{~m}$. Only the data with good quality are used for data analysis and in the EDR retrieval techniques. The data are not used in case of one of the following conditions:

1) the sonic anemometer is possibly in the wake of the tower (explained in section 3a);

2) the radar reflectivity factor is less than $-20 \mathrm{~dB} Z$ for the main or offset beam;

3) the backscattering is inhomogeneous for the three beams (one beam reflectivity is more than the average $\pm 3 \mathrm{~dB}$ ); or

4) less than $50 \%$ of the samples are usable for a given total sampling time.
In the presence of precipitation, the measured radar Doppler velocity represents the reflectivity weighted LOS velocities of the scatterers. An estimate for the air LOS velocity is then obtained by a correction for the radar mean Doppler terminal fall velocity (details in section 2a).

The resulting 3D wind speed retrieved from TARA for the first range cell is shown in Fig. 5, and the vertical wind velocity is shown in Fig. 6 . The figures show qualitatively that the air wind speed is well captured by TARA, but the remotely retrieved vertical wind velocity has biases of up to $3 \mathrm{~m} \mathrm{~s}^{-1}$. A bias in the radarbased retrieved vertical velocities is by itself not problematic for the estimation of EDR, which is based on the velocity fluctuations. However, if the bias in estimated vertical velocity fluctuates with time, then the estimate for standard deviation of vertical air velocities will be compromised. In Fig. 6, fluctuations in the estimated vertical wind velocity of about $2 \mathrm{~m} \mathrm{~s}^{-1}$ are shown for the study cases. Therefore, it can be expected that the EDR values from the VWVV technique are less accurate.

The biases in vertical velocity have a complex origin that differs from case to case, and could be explained by 


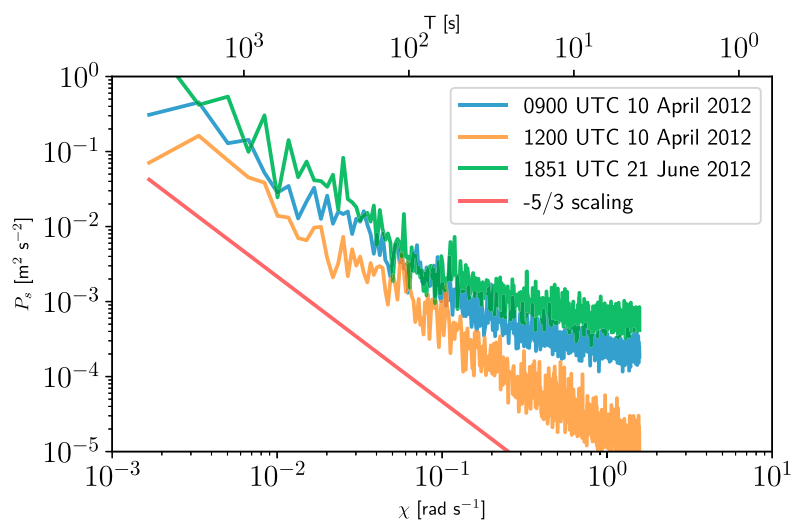

FIG. 8. Averaged power spectra of wind speed from the sonic anemometer. The discrete power $P_{k}\left(\mathrm{~m}^{2} \mathrm{~s}^{-2}\right)$ is plotted as a function of angular frequency $\chi\left(\mathrm{s}^{-1}\right)$. The averaging has been applied over 1000 spectra to reduce noise. The total sampling time was one hour $( \pm 3 \mathrm{~min}$ with respect to the time in the legend).

inaccurately estimated rain DSD parameters. To our knowledge, there has been no validation of radarbased estimated vertical air velocities during rain, which includes the estimation of DSD parameters. Note that for the case studies, the used correction for terminal fall velocity was still useful, because without such a correction the biases could be as large as the largest terminal fall speeds, which are up to about $10 \mathrm{~m} \mathrm{~s}^{-1}$.

\section{d. Comparison of retrieved EDR values}

One concern with the application of EDR retrieval techniques is whether the measurements are in the inertial range. Or with other words, Eq. (2) applies to the measurements. To analyze this, averaged power spectra are obtained from sonic anemometer wind speed measurements at $180-\mathrm{m}$ altitude for a few times points, and are shown in Fig. 8. We note here that the radar is not useful for estimating power spectra, because of the much longer sampling time and the radar data quality. Figure 8 shows that for short time scales (smaller than approximately $10^{2} \mathrm{~s}$, or approximately $\chi>10^{-1} \mathrm{rad} \mathrm{s}^{-1}$ ), the power spectra do not satisfy the $-5 / 3$ power scaling for 0900 and 1200 UTC 10 April 2012. This can be associated with boundary layer processes, such as energy transport, surface friction, and wind shear (see Fig. 5). The part of the power spectra of $3 \mathrm{D}$ wind speeds for time scales longer than $10^{2} \mathrm{~s}$ is in the inertial range. This proves that the velocity measurements at the scale of $10 \mathrm{~min}$ are in the inertial range. As a result, the assumption of measuring in the inertial range is demonstrated for the EDR retrieval techniques that are based on the energy estimate of velocities at the scale of $10 \mathrm{~min}$ in this work (WSV, VWVV). For the STWSV technique, the measurements will not always be in the inertial range, for which biased EDR estimates can be expected. For the techniques that process spectra of $3 \mathrm{D}$ wind speeds (PS, SSF), EDR values will be biased if the measurements are not in the inertial range, but at the same time their error estimate gives a measure of how well the measurements are in the inertial range.

Estimated EDR values and uncertainties are shown in Fig. 9 for both the TARA radar and the in situ measurements from the sonic anemometer. These EDR estimations are for the same times as the estimated power spectra in Fig. 8. For 1200 UTC 10 April 2012, consistency is found for the different EDR retrieval techniques, which can be expected as the obtained spectra in Fig. 8 showed that the inertial range was applicable for time scales from $\sim 10^{1}$ until $\sim 10^{3} \mathrm{~s}$. For the PS technique, rather large errors are found, which may be a consequence of insufficient averaging of spectra. We further note that the estimated uncertainty with error propagation (VWVV, WSV, and STWSV in Fig. 9) is often on the low side. This may be a consequence of that this estimated uncertainty does not include a broader range of the energy spectrum, which the other uncertainty estimates do (PS, SSF). In contrast to the PS technique, the error estimated with the SSF technique does not suffer from insufficient samples or averaging, and thus seems to be the best choice for uncertainty estimation from the discussed techniques.

In Figs. 10a and 10b, time series of EDR are shown for EDR retrieval techniques applied to in situ measurements from the sonic anemometer at $180-\mathrm{m}$ altitude. Both cases have values of EDR varying from approximately $10^{-7}$ up to $10^{-2} \mathrm{~m}^{2} \mathrm{~s}^{-3}$. An agreement is found for the different EDR retrieval techniques (WSV, VWVV, SSF), where the time variation of EDR is similar. The EDR values do not differ much more than one order of magnitude.

Time series for the remote retrieval of EDR values during rain from the TARA instrument are shown in Figs. 10c and 10d. The differences in EDR values from the remote radar measurements during rain from different techniques are now, in contrast to the in situ measurements, larger and up to two orders of magnitude. The time variation of the SSF technique is very similar to the WSV technique in Fig. 10c, which is based on the same measurements of $3 \mathrm{D}$ wind speeds. In Fig. 10d the SSF could not be applied, because the radar measurements contained incomplete time series, because of insufficient quality (see quality conditions in section $3 c$ ), which makes this technique less versatile in its application. The times series from the PS and STWSV techniques are not shown in Fig. 10, because of its large biases and uncertainties (see Fig. 9). 


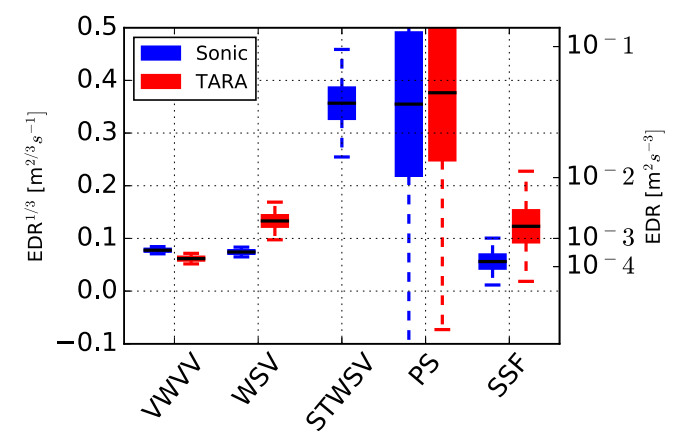

(a) 0900 UTC 10 April 2012

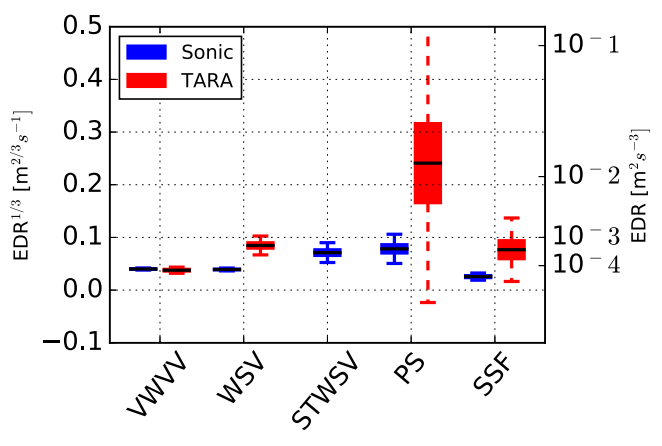

(b) 1200 UTC 10 April 2012

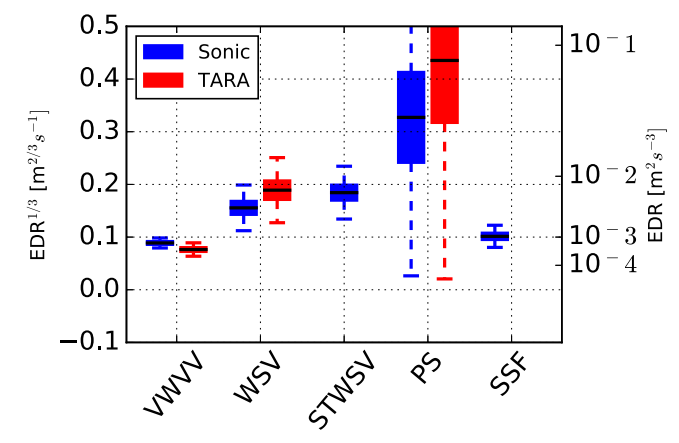

(c) 1851 UTC 21 June 2012

FIG. 9. Estimated EDR values and uncertainties for the first range cell of TARA (173- to 202-m altitude) and the sonic anemometer (180-m altitude) on: (a) 0900 UTC 10 Apr, (b) 1200 UTC 10 Apr, and (c) 1851 UTC 21 Jun 2012. The box indicates the standard deviation (STD) uncertainty and the outer edges 2 times the STD. For TARA the SSF could not be applied on 1851 UTC, because of insufficient samples.

In Fig. 11 scatter density plots are shown for the VWVV and WSV techniques to assess these techniques quantitatively. A correlation of 0.79 was found for the comparison of in situ versus radar-based logarithmic EDR values for the WSV technique during rain, which demonstrates a strong relationship. The retrieved values from the TARA radar are, however, biased toward higher EDR values $(+0.838$ for logarithmic EDR values): the sonic values logarithmic EDR values are predominantly between $10^{-4}$ and $10^{-3} \mathrm{~m}^{2} \mathrm{~s}^{-3}$, and the TARA radar logarithmic EDR values are predominantly between $10^{-3.5}$ and $10^{-2.5} \mathrm{~m}^{2} \mathrm{~s}^{-3}$. In addition to this bias, EDR values lower than $10^{-4.5} \mathrm{~m}^{2} \mathrm{~s}^{-3}$ were not found for the WSV technique when applied to the TARA radar. For the comparison of in situ versus radarbased logarithmic EDR values during rain for the VWVV during rain, a correlation of -0.20 was found, which demonstrates no relationship. This is most likely the result of unreliable raindrop terminal fall velocity corrections. The WSV is thus recommended for applications, but caution is necessary because very low EDR values cannot be estimated, and a bias toward higher EDR values should be expected. In future applications, it is expected that the estimation of low EDR values can be improved by reducing the error in the wind velocity standard deviation $\sigma_{\text {err }}$ [see Eq. (20)], by having an improved radar Doppler resolution and a higher signalto-noise ratio.

\section{Conclusions}

In this article, the goal was to find the best EDR retrieval technique-from five typically applied EDR retrieval techniques-for the Doppler radar that is capable to measure EDR under rainy conditions. A correction for the raindrop terminal fall speed was applied. The best (out of five) EDR retrieval technique for the Doppler radar was determined to be the wind speed variance (WSV) technique by comparing retrieved EDR values based on estimated wind velocities from the TARA radar during rain and collocated EDR values from a sonic anemometer on a tower lever at 180-m altitude.

One concern with the retrieval of EDR values is whether the measurements are in the inertial subrange. To verify this, power spectra of $3 \mathrm{D}$ wind speeds from a 


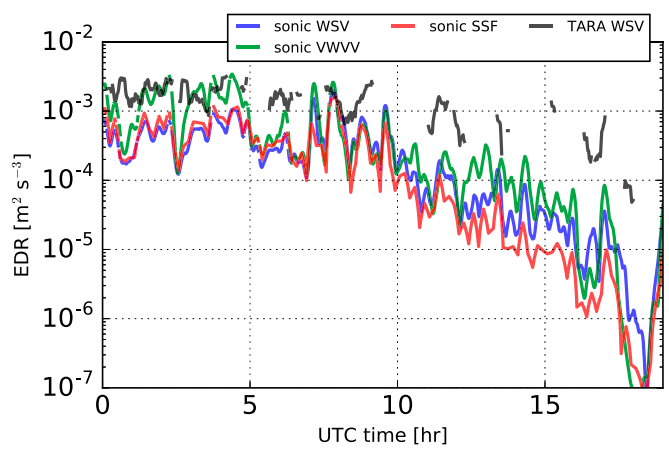

(a)

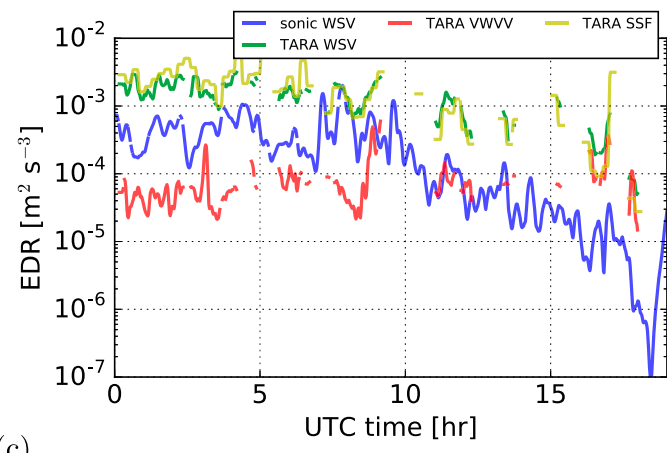

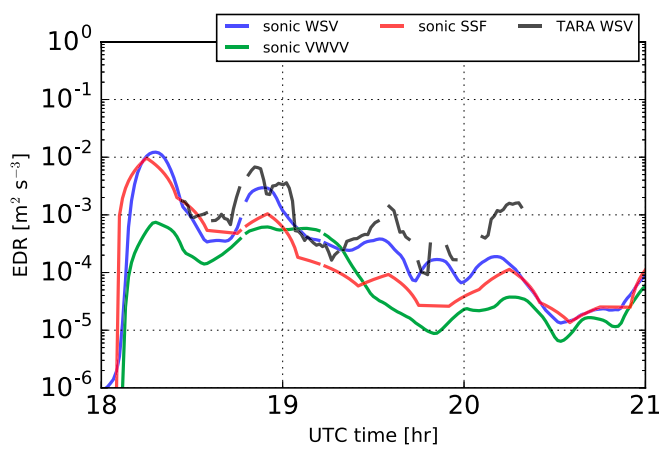

(b)

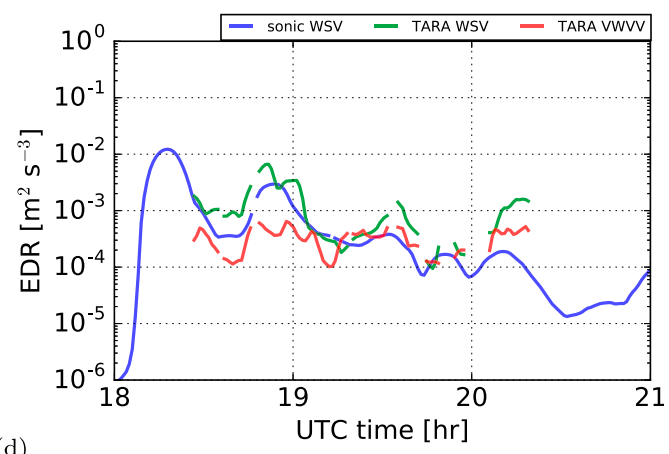

(d)

FIG. 10. Retrieved EDR values from the sonic anemometer and the TARA radar for (left) 10 Apr and (right) 21 June 2012. (top) Results from a set of EDR retrieval techniques are shown, which were obtained from the sonic anemometer at 180-m altitude. (bottom) Results from a set of EDR retrieval techniques applied to the first range cell of TARA (173- to 202-m altitude) are shown. For comparison, the WSV technique is shown for both instruments in all these plots.

sonic anemometer at $180-\mathrm{m}$ altitude were analyzed, and with them it was demonstrated that for the case studies there was always an inertial range of time scales between $10^{2}$ and $10^{3} \mathrm{~s}$. Therefore, the EDR retrieval techniques that use a time scale of $10 \mathrm{~min}$ in this work were applied in the inertial range. These techniques are: the wind speed variance (WSV) and the vertical wind velocity variance (VWVV) EDR retrieval techniques. For techniques that

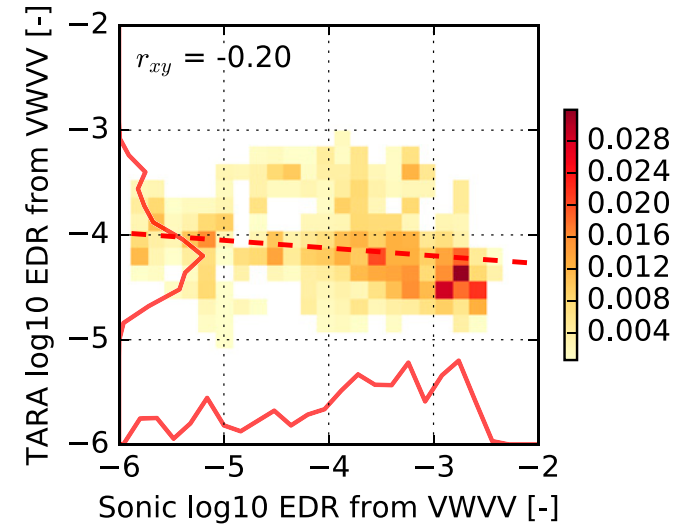

(a) VWVV

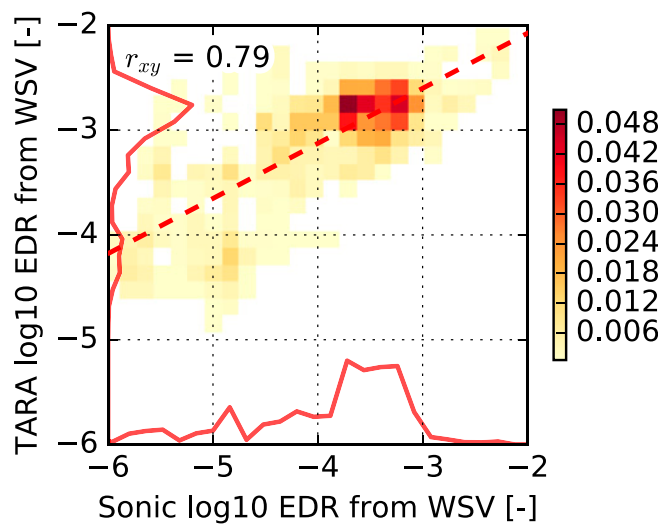

(b) WSV

FIG. 11. Scatter density plot of EDR values obtained with (a) VWVV and (b) WSV retrieval techniques for the two case studies. Relative occurrence is plotted in each pixel for the radar ( $y$ axis) against the in situ sonic measurements ( $x$ axis). On top of the axis the relative distribution is plotted and the correlation coefficient $r_{x y}$ is printed. 
were applied at a shorter time scale or take a spectrum of times scales into account in this work EDR values could be biased, because they may be applied outside the inertial range. These techniques are the power spectrum (PS), the second-order structure function (SSF), and the short time wind speed variance (STWSV) EDR retrieval techniques. The estimated uncertainty from the processing of 3D wind speed spectra (SSF, PS) can be useful for these cases, as it quantifies how well the measurements are in the inertial range.

Two rain events on 10 April and on 21 June 2012 were used to demonstrate and assess the EDR retrieval techniques. For these case studies, an agreement was found for EDR retrieval techniques (WSV, VWVV, SSF), when they were applied to the sonic anemometer. Other techniques were biased, likely because of large errors (PS), or the application to a short time scale (STWSV). When the EDR retrieval techniques are applied to Doppler velocity measurements from the radar during rain, the resulting EDR values are rather different in comparison with in situ-based EDR values. A correlation coefficient of 0.79 was found for radarbased retrieved logarithmic EDR values and in situbased retrieved logarithmic EDR values for the WSV EDR retrieval technique, which indicates a strong relationship and is considered the best. The VWVV EDR retrieval technique, which is often applied to lidar and sonic anemometer measurements, is less effective for the radar during rain, because the radar-based estimated vertical velocities were not sufficiently accurate. Although the technique based on the SSF can be useful, in particular for a reliable uncertainty estimation that is based on a broad range of time scales, its application to Doppler radar measurements is limited because of incomplete time series due to noncontinuous beam filling.

The conclusions in this article are based on two case studies that were done in Cabauw, the Netherlands, where radar-based EDR values were compared with in situ-based EDR values by using a sonic anemometer that was located on a meteorological tower. For further applications to other Doppler radars during rain, it is recommended that the concept of EDR is applied cautiously. A minimal retrievable EDR should be estimated for the used instrument, and for each EDR value uncertainties should be provided. In addition, it is suggested to always provide the sampling and total sampling scales that were used in the EDR retrieval technique. Also, the estimation of energy spectra of 3D wind speeds is advised, which can be used to validate if the measurements were taken in the inertial range. With regard to the radar-based drop size distribution (DSD) estimation, not only the DSD parameters should be verified, but also the associated vertical air velocities. The radar-based estimated vertical air velocities can be verified by doing a comparison of the 10-min-averaged estimated vertical air velocity with values from an independent source such as in situ sonic anemometer measurements or a numerical weather model. Further, it is expected that these retrieval techniques can also be applied - with some modificationsto radar-based EDR retrievals during snow and hail. In all of the future studies, long-term and consistent observations of EDR are recommended, which leads to testing of the algorithms under sufficiently various weather conditions.

Acknowledgments. This work has been done in the framework of the EU FP7 program, the UFO project. In addition to that we acknowledge the usage of sonic anemometer data from the CESAR database. It has to be said that observatories with long-term data records are struggling to survive politically motivated financial cuts but are essential for the validation of remote sensing applications, such as the validation of EDR retrieval techniques.

\section{REFERENCES}

Albrecht, B., M. Fang, and V. Ghate, 2016: Exploring stratocumulus cloud-top entrainment processes and parameterizations by using Doppler cloud radar observations. J. Atmos. Sci., 73, 729-742, https://doi.org/10.1175/JAS-D-15-0147.1.

Anagnostou, E. N., M. N. Anagnostou, W. F. Krajewski, A. Kruger, and B. J. Miriovsky, 2004: High-resolution rainfall estimation from X-band polarimetric radar measurements. J. Hydrometeor., 5, 110-128, https://doi.org/10.1175/15257541(2004)005<0110:HREFXP $>2.0$.CO;2.

Atlas, D., R. C. Srivastava, and R. S. Sekhon, 1973: Doppler radar characteristics of precipitation at vertical incidence. Rev. Geophys., 11, 1-35, https://doi.org/10.1029/RG011i001p00001.

Barbaresco, F., and Coauthors, 2013: Wake vortex detection, prediction and decision support tools in SESAR program. 32nd Digital Avionics Systems Conf., East Syracuse, NY, IEEE, 6B1-1-6B1-15, https://doi.org/10.1109/DASC.2013.6712614.

, and Coauthors, 2014: Radar 3D monitoring of wake-vortex hazards, circulation and EDR retrieval/calibration. Int. Radar Conf., 1-7, Lille, France, IEEE, 10.1109/RADAR.2014.7060419.

_ B. Vincent, and J. Nicolas, 2016: Radar wake-vortices crosssection/Doppler signature characterisation based on simulation and field tests trials. IET Radar Sonar Navig., 10, 82-96, https://doi.org/10.1049/iet-rsn.2015.0132.

Borque, P., E. Luke, and P. Kollias, 2016: On the unified estimation of turbulence eddy dissipation rate using Doppler cloud radars and lidars. J. Geophys. Res. Atmos., 121, 5972-5989, https:// doi.org/10.1002/2015JD024543.

Bouniol, D., A. Illingworth, and R. Hogan, 2004: Deriving turbulent kinetic energy dissipation rate within clouds using ground based radar. Third European Conf. on Radar in Meteorology and Hydrology, Visby, Sweden, Copernicus, 281-285.

Boutle, I. A., and S. J. Abel, 2012: Microphysical controls on the stratocumulus topped boundary-layer structure during VOCALS-REx. Atmos. Chem. Phys., 12, 2849-2863, https:// doi.org/10.5194/acp-12-2849-2012. 
Brandes, E. A., G. Zhang, and J. Vivekanandan, 2004: Comparison of polarimetric radar drop size distribution retrieval algorithms. J. Atmos. Oceanic Technol., 21, 584-598, https://doi.org/10.1175/ 1520-0426(2004)021<0584:COPRDS > 2.0.CO;2.

Bringi, V. N., and V. Chandrasekar, 2001: Polarimetric Doppler Weather Radar. Cambridge University Press, $636 \mathrm{pp}$.

Brost, R. A., J. C. Wyngaard, and D. H. Lenschow, 1982: Marine stratocumulus layers. Part II: Turbulence budgets. J. Atmos. Sci., 39, 818-836, https://doi.org/10.1175/1520-0469(1982) 039<0818:MSLPIT > 2.0.CO;2.

Bryant, G. W., and K. A. Browning, 1975: Multi-level measurements of turbulence over the sea during the passage of a frontal zone. Quart. J. Roy. Meteor. Soc., 101, 35-54, https:// doi.org/10.1002/qj.49710142705.

Casso-Torralba, P., J. V. G. de Arellano, F. Bosveld, M. R. Soler, A. Vermeulen, C. Werner, and E. Moors, 2008: Diurnal and vertical variability of the sensible heat and carbon dioxide budgets in the atmospheric surface layer. J. Geophys. Res., 113, D12119, https://doi.org/10.1029/2007JD009583.

Caughey, S. J., J. C. Wyngaard, and J. C. Kaimal, 1979: Turbulence in the evolving stable boundary layer. J. Atmos. Sci., 36, 1041-1052, https://doi.org/10.1175/1520-0469(1979)036<1041: TITESB $>2.0 . \mathrm{CO} ; 2$.

Chan, P. W., 2011: Generation of an eddy dissipation rate map at the Hong Kong International Airport based on Doppler lidar data. J. Atmos. Oceanic Technol., 28, 37-49, https://doi.org/ 10.1175/2010JTECHA1458.1.

Doviak, R. J., and D. S. Zrnić, 1993: Doppler Radar and Weather Observations. 2nd ed. Academic Press, 562 pp.

Fang, M., B. A. Albrecht, V. P. Ghate, and P. Kollias, 2014: Turbulence in continental stratocumulus. Part I: External forcings and turbulence structures. Bound.-Layer Meteor., 150, 341360, https://doi.org/10.1007/s10546-013-9873-3.

Frech, M., 2007: Estimating the turbulent energy dissipation rate in an airport environment. Bound.-Layer Meteor., 123, 385-393, https://doi.org/10.1007/s10546-006-9149-2.

Garratt, J. R., 1992: The Atmospheric Boundary Layer. Cambridge Atmospheric and Space Science Series, Cambridge University Press, 336 pp.

Gerz, T., F. Holzäpfel, W. Bryant, F. Köpp, M. Frech, A. Tafferner, and G. Winckelmans, 2005: Research towards a wake-vortex advisory system for optimal aircraft spacing. C. R. Phys., 6, 501-523, https://doi.org/10.1016/j.crhy.2005.06.002.

Gossard, E. E., 1990: Radar research on the atmospheric boundary layer. Radar in Meteorology, D. Atlas, Ed., Springer, 477-527.

Heijnen, S., L. Ligthart, and H. Russchenberg, 2000: First measurements with TARA; an S-band transportable atmospheric radar. Phys. Chem. Earth, 25B, 995-998, https://doi.org/ 10.1016/S1464-1909(00)00140-4.

Holzäpfel, F., 2006: Probabilistic two-phase aircraft wake-vortex model: Further development and assessment. J. Aircr., 43, 700-708, https://doi.org/10.2514/1.16798.

_, T. Gerz, F. Köpp, E. Stumpf, M. Harris, R. I. Young, and A. Dolfi-Bouteyre, 2003: Strategies for circulation evaluation of aircraft wake vortices measured by lidar. J. Atmos. Oceanic Technol., 20, 1183-1195, https://doi.org/10.1175/1520-0426(2003) $020<1183:$ SFCEOA $>2.0 . \mathrm{CO} ; 2$.

ICAO, 2007: Annex 3 to the Convention on International Civil Aviation. International Civil Aviation Organization Tech. Rep., 67 pp.

Kaimal, J. C., J. C. Wyngaard, D. A. Haugen, O. R. Coté, Y. Izumi, S. J. Caughey, and C. J. Readings, 1976: Turbulence structure in the convective boundary layer. J. Atmos. Sci., 33, 2152-2169,
https://doi.org/10.1175/1520-0469(1976)033<2152:TSITCB > 2.0.CO;2.

Katul, G. G., 1994: A model for sensible heat flux probability density function for near-neutral and slightly-stable atmospheric flows. Bound.-Layer Meteor., 71, 1-20, https://doi.org/ 10.1007/BF00709217.

Khain, A. P., and Coauthors, 2015: Representation of microphysical processes in cloud-resolving models: Spectral (bin) microphysics versus bulk parameterization. Rev. Geophys., 53, 247-322, https://doi.org/10.1002/2014RG000468.

Kollias, P., and B. Albrecht, 2000: The turbulence structure in a continental stratocumulus cloud from millimeter-wavelength radar observations. J. Atmos. Sci., 57, 2417-2434, https://doi.org/ 10.1175/1520-0469(2000)057<2417:TTSIAC >2.0.CO;2.

,$- \ldots$, R. Lhermitte, and A. Savtchenko, 2001: Radar observations of updrafts, downdrafts, and turbulence in fairweather cumuli. J. Atmos. Sci., 58, 1750-1766, https://doi.org/ 10.1175/1520-0469(2001)058<1750:ROOUDA > 2.0.CO;2.

Kolmogorov, A., 1941: Dissipation of energy in locally isotropic turbulence. Dokl. Akad. Nauk SSSR, 32, 16-18.

Kovalev, D., D. Vanhoenacker-Janvier, R. Wilson, and F. Barbaresco, 2016: Electromagnetic wind radar simulator validation using meteorological data and a zenith X-band radar. European Radar Conf., London, United Kingdom, IEEE, http://ieeexplore.ieee.org/ stamp/stamp.jsp?tp $=$ \&arnumber $=7811662 \&$ isnumber $=7811617$.

Lamer, K., and P. Kollias, 2015: Observations of fair-weather cumuli over land: Dynamical factors controlling cloud size and cover. Geophys. Res. Lett., 42, 8693-8701, https://doi.org/ 10.1002/2015GL064534.

Lane, J. E., T. Kasparis, P. T. Metzger, and W. L. Jones, 2014: In situ disdrometer calibration using multiple DSD moments. Acta Geophys., 62, 1450-1477, https://doi.org/10.2478/s11600014-0237-2.

Lemone, M. A., and W. T. Pennell, 1979: A comparison of turbulence measurements from aircraft. J. Appl. Meteor., 19, 1420-1437, https://doi.org/10.1175/1520-0450(1980)019<1420:ACOTMF $>$ 2.0.CO;2.

MacCready, P. B. J., 1964: Standardization of gustiness values from aircraft. J. Appl. Meteor., 3, 439-449, https://doi.org/10.1175/ 1520-0450(1964)003<0439:SOGVFA > 2.0.CO;2.

Marshall, J. S., and W. M. K. Palmer, 1948: The distribution of raindrops with size. J. Meteor., 5, 165-166, https://doi.org/ 10.1175/1520-0469(1948)005<0165:TDORWS > 2.0.CO;2.

Meagher, J. P., and Z. S. Haddad, 2006: To what extent can raindrop size be determined by a multiple-frequency radar? J. Appl. Meteor. Climatol., 45, 529-536, https://doi.org/ 10.1175/JAM2344.1.

Meischner, P., R. Baumann, H. Höller, and T. Jank, 2001: Eddy dissipation rates in thunderstorms estimated by Doppler radar in relation to aircraft in situ measurements. J. Atmos. Oceanic Technol., 18, 1609-1627, https://doi.org/10.1175/1520-0426(2001) 018<1609:EDRITE > 2.0.CO;2.

Meneveau, C., and J. Katz, 2000: Scale-invariance and turbulence models for large-eddy simulation. Annu. Rev. Fluid Mech., 32, 1-32, https://doi.org/10.1146/annurev.fluid.32.1.1.

Mishchenko, M., L. Travis, and A. Lacis, 2002: Scattering, Absorption, and Emission of Light by Small Particles. Cambridge University Press, 445 pp.

Nastrom, G. D., 1997: Doppler radar spectral width broadening due to beamwidth and wind shear. Ann. Geophys., 15, 786796, https://doi.org/10.1007/s00585-997-0786-7.

- and F. D. Eaton, 1997: Turbulence eddy dissipation rates from radar observations at $5-20 \mathrm{~km}$ at White Sands Missile 
Range, New Mexico. J. Geophys. Res., 102, 19495-19505, https://doi.org/10.1029/97JD01262.

Nicholls, S., 1978: Measurements of turbulence by an instrumented aircraft in a convective atmospheric boundary layer over the sea. Quart. J. Roy. Meteor. Soc., 104, 653-676, https://doi.org/ 10.1002/qj.49710444109.

Nucciarone, J. J., and G. S. Young, 1991: Aircraft measurements of turbulence spectra in the marine stratocumulus-topped boundary layer. J. Atmos. Sci., 48, 2382-2392, https://doi.org/ 10.1175/1520-0469(1991)048<2382:AMOTSI >2.0.CO;2.

O'Connor, E. J., A. J. Illingworth, I. M. Brooks, C. D. Westbrook, R. J. Hogan, F. Davies, and B. J. Brooks, 2010: A method for estimating the turbulent kinetic energy dissipation rate from a vertically pointing Doppler lidar, and independent evaluation from balloon-borne in situ measurements. J. Atmos. Oceanic Technol., 27, 1652-1664, https://doi.org/10.1175/2010JTECHA1455.1.

Oude Nijhuis, A. C. P., F. J. Yanovsky, O. Krasnov, C. M. Unal, H. W. Russchenberg, and A. Yarovoy, 2016: Assessment of the rain drop inertia effect for radar-based turbulence intensity retrievals. Int. J. Microwave Wireless Technol., 8, 835844, https://doi.org/10.1017/S1759078716000660.

— , and Coauthors, 2018: Wind hazard and turbulence monitoring at airports with lidar, radar, and Mode-S downlinks: The UFO Project. Bull. Amer. Meteor. Soc., 99, 2275-2293, https:// doi.org/10.1175/BAMS-D-15-00295.1.

Piper, M., and J. K. Lundquist, 2004: Surface layer turbulence measurements during a frontal passage. J. Atmos. Sci., 61, 1768-1780, https://doi.org/10.1175/1520-0469(2004)061<1768: SLTMDA $>2.0 . \mathrm{CO} ; 2$.

Pope, S. B., 2000: Turbulent Flows. Cambridge University Press, $801 \mathrm{pp}$.

Röhner, L., and K. Träumner, 2013: Aspects of convective boundary layer turbulence measured by a dual-Doppler lidar system. J. Atmos. Oceanic Technol., 30, 2132-2142, https:// doi.org/10.1175/JTECH-D-12-00193.1.

Shupe, M. D., I. M. Brooks, and G. Canut, 2012: Evaluation of turbulent dissipation rate retrievals from Doppler cloud radar. Atmos. Meas. Tech., 5, 1375-1385, https://doi.org/10.5194/ amt-5-1375-2012.

Siebert, H., K. Lehmann, and M. Wendisch, 2006: Observations of small-scale turbulence and energy dissipation rates in the cloudy boundary layer. J. Atmos. Sci., 63, 1451-1466, https:// doi.org/10.1175/JAS3687.1.

Sutton, O. G., 1953: Micrometeorology: A Study of Physical Processes in the Lowest Layers of the Earth's Atmosphere. McGraw-Hill, 333 pp.
Taylor, G. I., 1938: The spectrum of turbulence. Proc. Roy. Soc. London, 164A, 476-490, https://doi.org/10.1098/ rspa.1938.0032.

Taylor, J. R., 1997: An Introduction to Error Analysis: The Study of Uncertainties in Physical Measurements. 2nd ed. University Science Books, 327 pp.

Thiermann, V., and H. Grassl, 1992: The measurement of turbulent surface-layer fluxes by use of bichromatic scintillation. Bound.-Layer Meteor., 58, 367-389, https://doi.org/10.1007/ BF00120238.

Thobois, L. P., R. Krishnamurty, J.-P. Cariou, A. Dolfi-Bouteyre, and M. Valla, 2015: Wind and EDR measurements with scanning Doppler lidars for preparing future weather dependent separation concepts. 7th Atmospheric and Space Environments Conf., Dallas, TX, American Institute of Aeronautics and Astronautics, https://doi.org/10.2514/ 6.2015-3317.

Unal, C., 2015: High-resolution raindrop size distribution retrieval based on the Doppler spectrum in the case of slant profiling radar. J. Atmos. Oceanic Technol., 32, 1191-1208, https:// doi.org/10.1175/JTECH-D-13-00225.1.

— , and D. N. Moisseev, 2004: Combined Doppler and polarimetric radar measurements: Correction for spectrum aliasing and nonsimultaneous polarimetric measurements. J. Atmos. Oceanic Technol., 21, 443-456, https://doi.org/10.1175/15200426(2004)021<0443:CDAPRM > 2.0.CO;2.

, Y. Dufournet, T. Otto, and H. Russchenberg, 2012: The new real-time measurement capabilities of the profiling TARA radar. Seventh European Conf. on Radar in Meteorology and Hydrology, Toulouse, France, Meteo France, 199 SP.

Van Ulden, A. P., and J. Wieringa, 1996: Atmospheric boundary layer research at Cabauw. Bound.-Layer Meteor., 78, 39-69, https://doi.org/10.1007/BF00122486.

White, A. B., R. J. Lataitis, and R. S. Lawrence, 1999: Space and time filtering of remotely sensed velocity turbulence. J. Atmos. Oceanic Technol., 16, 1967-1972, https://doi.org/10.1175/15200426(1999)016<1967:SATFOR > 2.0.CO;2.

Wilson, R., 2004: Turbulent diffusivity in the free atmosphere inferred from MST radar measurements: A review. Ann. Geophys., 22, 3869-3887, https://doi.org/10.5194/angeo-223869-2004.

Zhou, M. Y., D. H. Lenschow, B. B. Stankov, J. C. Kaimal, and J. E. Gaynor, 1985: Wave and turbulence structure in a shallow baroclinic convective boundary layer and overlying inversion. J. Atmos. Sci., 42, 47-57, https://doi.org/10.1175/15200469(1985)042<0047:WATSIA $>2.0$. CO;2. 